\title{
THE MATHEMATICAL THEORY OF RESONANCES WHOSE WIDTHS ARE EXPONENTIALLY SMALL
}

\author{
E. HARRELL AND B. SIMON
}

\section{Contents}

1. Introduction . . . . . . . . . . . . . . . . . . . .845

2. The Basic Strategy . . . . . . . . . . . . . . . . . . . . . 854

3. The Short-Range Case: A Warm-Up . . . . . . . . . . . . . . 860

4. The Titchmarsh Problem . . . . . . . . . . . . . . . . . . . 871

5. The Bender-Wu Formula . . . . . . . . . . . . . . . . . 880

6. The Oppenheimer Formula . . . . . . . . . . . . . . . . . . 883

Appendix A. Calculation of the Tunneling Factor . . . . . . . .888

Appendix B. Some Properties of Special Functions . . . . . . . . . 890

Appendix C. Construction of the Comparison Functions . . . . . . . 895

References . . . . . . . . . . . . . . . . . . . . . . . 9900

\$1. Introduction. Among the most interesting and challenging mathematical problems raised by non-relativistic quantum mechanics are those connected with resonances. This is due both to their subtle nature and to their practical importance. Let us begin by describing two situations which are typical of rather distinct general classes of examples; the first was completely analyzed by one of us [49] several years ago (relying, in part, on earlier work of Friedrichs and Howland; see [49] for references) - the second will be analyzed in this paper.

Example 0. Autoionizing states in helium. Let $A_{0}$ be the Schrödinger operator

$$
A_{0}=-\Delta_{1}-\Delta_{2}-\frac{2}{r_{1}}-\frac{2}{r_{2}}
$$

on $L^{2}\left(\mathbf{R}^{6}\right)$, where $\mathbf{r}$ in $\mathbf{R}^{6}$ is written $\left\langle\mathbf{r}_{1}, \mathbf{r}_{2}\right\rangle$, and $\Delta_{i}$ is the Laplacean in $\mathbf{r}_{i}$. Let

$$
A(\lambda)=A_{0}+\lambda\left|\mathbf{r}_{1}-\mathbf{r}_{2}\right|^{-1}
$$

( $A_{0}$ and $A(\lambda)$ are self-adjoint with domains taken as the Sobolev space $H_{2}$ ). For $\lambda=1, A_{0}$ is a very good approximation to the energy operator of the helium atom (and for $\lambda=(2 / Z)^{2}$, to a constant multiple of the energy operator of an ion consisting of a nucleus of charge $Z$ and two electrons). The continuous spectrum

Received March 29, 1980. Research partially supported by USNSF grant MCS 78-01885. Part of this work was done while E. Harrell held a NSF National Needs Fellowship at the Department of Mathematics, M.I.T. 
of $A_{0}$ is the interval $[-1, \infty)$, and many of its eigenvalues are imbedded in this continuous spectrum. For good mathematical and physical reasons one expects that these imbedded eigenvalues are not present for $A(\lambda)$ when $\lambda$ is small, but for the physical, observed case $A(1)$ one typically sees bumps in physical excitation spectra near the positions of the imbedded eigenvalues of $A_{0}$. In [49] (see also [39] for a pedagogical presentation), for each eigenvalue $E_{0}$ of $A_{0}$ a function $E(\lambda)$, analytic near zero, was found so that for $\phi$ Gaussian the only pole of $\left(\phi,(A(\lambda)-E)^{-1} \phi\right)$ near $E_{0}$ after continuation from $\operatorname{Im} E>0$ occurs at $E(\lambda)$. $E(\lambda)$ was interpreted as a "resonance" and $\Gamma(\lambda)=-2 \operatorname{Im} E(\lambda)$ is the resonance width. In general

$$
\Gamma(\lambda)=a_{2} \lambda^{2}+0\left(\lambda^{3}\right)
$$

with $a_{2}$ computable.

Example 1. The Stark effect in hydrogen. Here

$$
H_{0}=-\Delta-\frac{1}{r}
$$

is the Hamiltonian on $L^{2}\left(\mathrm{R}^{3}\right)$ of a hydrogen atom, and

$$
H(F)=H_{0}-F z
$$

(with $z$ the third component of $\mathbf{r}$ ) is the Hamiltonian in a constant electric field $(0,0, F) . H_{0}$ has the familiar Bohr spectrum: $[0, \infty)$ as continuous spectrum and eigenvalues at $-1 / 4 n^{2}$. The basic spectral properties of $H(F)$ for $F \neq 0$ have been mainly known since the work of Titchmarsh [54] (see [2, 23, 56, 50,57] for significant recent related work): $H(F)$ has $(-\infty, \infty)$ as spectrum, no eigenvalues, and purely absolutely continuous spectrum. Here, too, one expects there to be resonance energy functions $E(F)$ with $E(F) \rightarrow E_{0}$ as $F \rightarrow 0$. There is, however, a big difference between this problem and the last, namely that $\Gamma$ is exponentially small. Schrödinger [41] originally developed his perturbation series to treat the Hamiltonian (1.3) assuming that $H(F)$ has actual eigenvalues $E(F)$ near $E_{0}$. His methods can be used to obtain formal series in the nondegenerate cases,

$$
E(F) \sim \sum_{n=0}^{\infty} A_{2 n} F^{2 n}
$$

with all the coefficients $A_{2 n}$ real (and finite; if one tries the analogous procedure in Example 0 one gets ill-defined integrals $\int\left(x-x_{0}\right)^{-1} g(x) d x$-the Taylor coefficients there can be obtained by systematically interpreting $\left(x-x_{0}\right)^{-1}$ as the distribution $\left.P\left(\left(x-x_{0}\right)^{-1}\right)-i \pi \delta\left(x-x_{0}\right)\right)$. Shortly thereafter, Oppenheimer [38] pointed out that because the electron could tunnel through the Coulombic potential barrier, $H(F)$ should not be expected to have stationary states, i.e., normalizable eigenvectors. Indeed, using formal, WKB-type formulae for the tunneling, he "computed" the width of the lowest resonance. A more systematic 
WKB calculation was then made by Lanczos [30]. There is some controversy $[58,59]$ about whether Oppenheimer's ideas lead to the correct answer even after correcting his algebraic mistakes; nevertheless, following standard terminology we call the "correct" WKB formula as found in Landau-Lifshitz [31] the Oppenheimer formula. It states that

$$
\Gamma(F) \sim \frac{1}{2} F^{-1} \exp \left(-\frac{1}{6 F}\right)
$$

for the width of the resonance associated with the lowest eigenvalue of $H_{0}$. One of our main goals in this paper will be to give a proof of this formula. Despite the fact that it was first "derived" fifty years ago there is not only no rigorous proof but even still some considerable discussion in the physical literature of its correctness. The reason for this and the associated reason for the complexity of the proof below is that one must be sure that every error term arising along the way is not merely small in the sense usual in perturbation analyses (i.e. $O\left(F^{k}\right)$ for some $k$ ), but small compared to the right side of (1.5). Indeed, although Yamabe et al. [58,59] (who also review much of the literature) have an approach with many similarities to ours, it is not at all clear how to control the errors without the various technical devices we use below.

For comparison with some of the literature, we note that an alternative normalization of (1.3) is

$$
H^{\prime}\left(F^{\prime}\right)=-\frac{1}{2} \Delta-\frac{1}{r}+F^{\prime} z
$$

Notice that under the scaling $\mathbf{r} \rightarrow-\frac{1}{2} \mathbf{r}, H^{\prime}\left(F^{\prime}\right)$ is unitary equivalent to $2 H\left(\frac{1}{4} F^{\prime}\right)$, so that

$$
\begin{aligned}
E^{\prime}\left(F^{\prime}\right) & =2 E\left(\frac{1}{4} F^{\prime}\right) \\
\Gamma^{\prime}\left(F^{\prime}\right) & =2 \Gamma\left(\frac{1}{4} F^{\prime}\right) \\
A_{2 n}^{\prime} & =2\left(\frac{1}{4}\right)^{2 n} A_{2 n} .
\end{aligned}
$$

Let us next say something about previous rigorous work on resonances in the Stark problem. Many of the ideas go back to Titchmarsh, both in his book [54] and in a series of papers [55], who worked on three interrelated approaches: spectral concentration, poles of Green functions, and "transmutation formulae."

Spectral concentration has been discussed and extended by a large number of authors; see [39] for complete references. We note that given Howland's ideas [27] on the connection between poles and spectral concentration and given our results below, one can considerably improve the spectral concentration results previously known for the Stark problem in hydrogen.

The connection between poles of Green functions and resonances has already been noted above. Titchmarsh studied them most especially in a model which is a simplification of the Stark problem: 
Example 2. (The Titchmarsh problem). Consider the Hamiltonian

$$
h(g, z, f)=-\frac{d^{2}}{d x^{2}}+\frac{g}{x^{2}}-\frac{z}{x}-f x
$$

with $f$ real, $g \geqslant-1 / 4$ and $z>0$ fixed parameters, on the space $L^{2}((0, \infty), d x)$. Questions about the boundary condition at zero will be discussed extensively below. $h$ arises if one reduces the $\nu$-dimensional Hamiltonian $-\Delta-z r^{-1}-f r$ to subspaces $\left\{r^{\nu / 2} u(r) Y_{\alpha}(\Omega)\right\}$ with $Y_{\alpha}$ a fixed spherical harmonic. For $\nu$ even the values of $g$ that occur are $g=m^{2}-1 / 4$ with $m=\frac{1}{2}(\nu-2), \frac{1}{2} \nu, \frac{1}{2}(\nu+2), \ldots$ and for $\nu$ odd, the values $g=l(l+1)$ with $l=\frac{1}{2}(\nu-3), \frac{1}{2}(\nu-1), \ldots$ In either event the quantity

$$
m \equiv(g+1 / 4)^{1 / 2}
$$

is always either integral or half-integral under this reduction. For these values of $m$, Titchmarsh $[54, \S 20.11]$ studied the problem for fixed $z$ and $m$ (or equivalently $g$ ), allowing $f$ to vary near zero. He proved that for any fixed eigenvalue $\lambda_{0}$ of the problem with $f=0$, the Green function $G(x, y ; \lambda)$ defined for $\operatorname{Im} \lambda>0$ has a meromorphic continuation in $\lambda$ for fixed $x$ and $y$, into a neighborhood of $\lambda=\lambda_{0}$, with a pole at a point $\lambda(f)$. He proved three facts about $\lambda(f)$ :

(i) $\lambda(f)=\lambda_{0}+\lambda^{(1)} f+O\left(f^{2} \ln (1 / f)\right)$

(ii) $\operatorname{Im} \lambda(f)=O\left(f^{2}\right)$

(iii) $\operatorname{Im} \lambda(f) \geqslant C_{1} \exp \left(-C_{2} f^{-1 / 4}\right), C_{1,2}>0$.

He conjectured that $\operatorname{Im} \lambda(f)$ was exponentially small, which is one of the things we prove in this paper. With our methods, or alternatively with those of Herbst [24] discussed below, one can establish an asymptotic series for $\lambda(f)$ valid to all orders, which improves (i); and the observation that all coefficients are real immediately improves (ii) to $O\left(f^{N}\right)$ for all $N<\infty$. See [20] for further discussion of this model.

Titchmarsh also considered these pole ideas in the context of the Stark effect. Because of the singularities of $G(\mathbf{x}, \mathbf{y} ; E)$ at $\mathbf{x}=\mathbf{y}$ and the corresponding failure of eigenfunction expansions to converge absolutely, Titchmarsh dealt instead with the kernel of the spectral projection $\int_{0}^{E} \operatorname{Im} G(\mathbf{x}, \mathbf{y} ; \mu+i O) d \mu$. Some related ideas have been developed by Schwinger [43] and Lovelace [37]. Most recent progress in the connection between poles and resonances originates in the work of Combes [10], who proposed looking for poles of $G$ when smeared in $\mathbf{x}, \mathbf{y}$ with vectors $\eta$ which are analytic for the group of dilations $\eta(\mathbf{x}) \rightarrow e^{\nu \theta / 2} \eta\left(e^{\theta} \mathbf{x}\right)$. In fact, our solution [49] of Problem 0 depended intimately on the development of these ideas by Balslev and Combes [3]. For some years it was clear that the Stark problem did not fit into the Balslev-Combes framework and there appeared to be severe formal problems preventing any extension. However, in a recent striking paper, Herbst [24] succeeded in extending the notions of [3] to allow the definition of a resonance; in particular, he was able to prove the occurrence of resonance poles in the smeared Green function for the Stark effect in hydrogen 
and he showed that the resonance functions $E(F)$ obey:

$$
E(F)=E_{0}+\sum_{n=1}^{N} A_{2 n} F^{2 n}+O\left(F^{2 N+2}\right)
$$

for the Rayleigh-Schrödinger coefficients $A_{2 n}$, which are all real.

$$
\begin{aligned}
\operatorname{Im} E(F)=O\left(F^{2 N}\right), & \text { all } N \\
-\operatorname{Im} E(F)>0, & \text { all } F \neq 0 .
\end{aligned}
$$

The quantity for which we prove Oppenheimer's formula (1.5) is precisely Herbst's $E(F)$.

Independently of Herbst, Graffi and Grecchi [18] used the transmutation formulae given below to discuss resonance poles for the Green function smeared with dilation-analytic vectors.

Herbst's methods have been extended by Herbst and Simon [26] to prove (i)-(ii) for multi-electron atoms in external electric fields. Unfortunately, as we shall explain later, our methods here are limited to the case of hydrogen. A major open question is the proof of generalized Oppenheimer formulae for multi-electron atoms.

There is a second set of problems, at first seemingly unrelated, that we will study in this paper, The canonical example of this problem is:

Example 3. (The anharmonic oscillator and the Bender-Wu formula). Let $\Lambda(\beta)$ denote the lowest eigenvalue of $Q(\beta)=-d^{2} / d v^{2}+v^{2}+\beta v^{4}$ on $L^{2}((-\infty$, $\infty) ; d v)$. By work of Titchmarsh [55] and Kato [29], it is known that $\Lambda(\beta)$ has an asymptotic series to all orders,

$$
\Lambda(\beta) \sim \sum_{n=0}^{\infty} a_{n} \beta^{n}
$$

By a numerical analysis of the first $75 a$ 's, which they computed by a simple recursive formula, Bender and $\mathrm{Wu}[5]$ deduced the asymptotic formula

$$
a_{n} \sim 4 \pi^{-3 / 2}(-1)^{n+1}\left(\frac{3}{2}\right)^{n+1 / 2} \Gamma\left(n+\frac{1}{2}\right), \quad(\text { as } n \rightarrow \infty)
$$

(they guessed the constant in front from a numerical 9-place decimal!). Several years later, they realized a connection between (1.9) and some WKB tunneling formulae [6]. To explain their idea, we must mention some analyticity results of Simon [48], Loeffel and Martin [35], and Loeffel et al. [36]: $\Lambda(\beta)$ has an analytic continuation to the complex plane cut along the negative real axis, with continuous boundary values. Moreover, $|\Lambda(\beta)| \leqslant A+B|\beta|^{1 / 3}$ in the whole plane. From these results and a Cauchy integral formula, one easily deduces [48] that for $n \geqslant 1$ :

$$
a_{n}=(-1)^{n+1}\left(\pi^{-1} \int_{0}^{\infty} \mu^{-n-1} \operatorname{Im} \Lambda(-\mu+i O) d \mu\right)
$$


Thus, the large $n$ asymptotics of $a_{n}$ follow from the small $\mu$ asymptotics of $\operatorname{Im} \Lambda(-\mu+i O)$. Bender and $\mathrm{Wu}$ [6] remarked that for $\mu>0$ and small, $-d^{2} / d x^{2}+x^{2}-\mu x^{4}$ is formally a tunneling problem, which they then analyzed using nonrigorous WKB formulae, finding that

$$
\operatorname{Im} \Lambda(-\mu+i O) \sim 4 \pi^{-1 / 2} \mu^{-1 / 2} \exp (-2 / 3 \mu)
$$

(Simon [48] had earlier noted that this would imply (1.9)). We will prove (1.11) below and thereby, given (1.10), also prove (1.9). Our methods should extend easily to the case of $x^{2 m}$ oscillators. The transmutation formulae do not hold in that case, but we only need them to reduce this operator to the Stark problem.

To facilitate comparison with the formulae of Bender and $\mathrm{Wu}$, we note that their normalization is

$$
Q^{\prime}\left(\beta^{\prime}\right)=-\frac{d^{2}}{d x^{2}}+\frac{1}{4} x^{2}+\frac{1}{4} \beta^{\prime} x^{4} .
$$

Under the scaling $x \rightarrow \sqrt{2} x, Q^{\prime}\left(\beta^{\prime}\right)$ is unitarily equivalent to $\frac{1}{2} Q\left(2 \beta^{\prime}\right)$. Hence

$$
\begin{aligned}
\Lambda^{\prime}\left(\beta^{\prime}\right) & =\frac{1}{2} \Lambda\left(2 \beta^{\prime}\right) \\
\operatorname{Im} \Lambda^{\prime}\left(-\mu^{\prime}+i O\right) & =\frac{1}{2} \operatorname{Im} \Lambda\left(-2 \mu^{\prime}+i O\right) \\
a_{n}^{\prime} & =2^{n-1} a_{n} .
\end{aligned}
$$

Parenthetically we note, following Herbst and Simon $[25,26]$, that the relation (1.10) has an analogue for the Stark effect, viz.,

$$
A_{2 n}=-\pi^{-1} \int_{0}^{R} F^{-2 n-1} \Gamma(F) d F+O\left(R^{-2 n}\right),
$$

with $A_{2 n}$ given by (1.4) and $\Gamma=-2 \operatorname{Im} E$. Thus, given our proof of (1.5) we also have an asymptotic formula for the $A_{2 n}$ perturbation coefficients for the Stark problem:

$$
A_{2 n} \sim-6^{2 n+1}(2 \pi)^{-1}(2 n) !
$$

Given the necessary changes of units, this agrees with numerical calculations of the $A_{2 n}[45,47]$.

We also note that considerable interest has been shown in (1.11) and (1.9) recently in terms of saddlepoint analysis in function space integrals (e.g., $[34,8])$. Despite considerable partial progress towards a rigorous justification of this approach (see [51,52]), (1.9) has not been proven by those means. Our proof of (1.9) sheds no light on why the formal function-space arguments give the right answer.

There is clearly a formal similarity between Problems 1-3 in that all involve tunneling through barriers and relations between imaginary parts of resonance 
energies and asymptotics of perturbation coefficients. Actually there is a closer similarity than that. The problems, at least after suitably imbedding them in problems with more parameters, are essentially identical. This realization is intimately connected with the separability of the Stark problem in suitable coordinates. Jacobi [28] seems to be the first to have studied the exact solution (up to quadratures) of the problem of a classical particle in an inverse-square force plus a constant external force field. He used the separability in elliptic coordinates. In 1916, Schwarzschild [42] and Epstein [13] independently reexamined the problem in order to explain the splitting of the Stark effect (observed by Stark [53] in 1913) within the old quantum theory of Bohr. Schwarzschild relied on Jacobi's solution, while Epstein introduced the separability in parabolic coordinates which, as we shall see, leads to coupled anharmonic oscillators. In 1926, Schrödinger [41] and Epstein [14] independently carried this separability in parabolic coordinates over to the "new" quantum mechanics, obtaining essentially the same basic transmutation formulae we describe below. This separation in parabolic coordinates is behind much of Titchmarsh's work [54,55] and also much of the recent work of the Modena group $[18,19,20,4]$. In particular, the essential equivalence of Oppenheimer's formula and the Bender-Wu formula (more properly the Bender-Wu-Banks formula discussed below) has been noted independently by Benassi and Grecchi [4].

To describe the equivalence we increase the number of free parameters. In Example 1, we define

$$
H(F, Z)=-\Delta-Z r^{-1}-F z
$$

and we write the formal eigenfunction equation

$$
H(F, Z) \Theta(\mathbf{r} ; Z, F)=E(Z, F) \Theta(\mathbf{r} ; Z, F) .
$$

In Example 2, we deal with the operator $h(g, z, f)$ and always let $m$ be given by (1.8). For $g \geqslant 3 / 4(m \geqslant 1), h$ is essentially self-adjoint on $C_{0}^{\infty}(0, \infty)$, and the condition of square-integrability at $x=0$ forces a boundary condition at $x=0$. For $-1 / 4 \leqslant g<3 / 4(0 \leqslant m<1)$, all solutions of the equation are squareintegrable at zero, behaving as $x^{\frac{1}{2} \pm m}$ (or, if $m=0$, as $x^{1 / 2}$ or $x^{1 / 2} \ln x$ ). If

$$
h(g, z, f) J(x ; g, z, f)=\lambda J(x ; g, z, f)
$$

with the boundary condition

$$
\varlimsup_{x \rightarrow 0} J(x) / x^{\frac{1}{2}+m}<\infty,
$$

then we say that $J$ obeys Dirichlet-like boundary conditions (for $g=0$, this corresponds precisely to Dirichlet boundary conditions; also, as we shall see, for $g=-3 / 16(m=1 / 4)$ a transmuted equation goes over to one with Dirichlet 
conditions). If on the other hand

$$
\lim _{x \rightarrow 0} x^{\frac{1}{2}-m}\left[J(x) / x^{\frac{1}{2}-m}\right]^{\prime}=\lim _{x \rightarrow 0}\left[J^{\prime}(x)-\left(\frac{1}{2}-m\right) J(x) / x\right]=0,
$$

then we say that $J$ obeys Neumann-like boundary conditions (again, for $g=0$, this means Neumann conditions, and for $g=-3 / 16$, Neumann conditions for the transmuted problem).

Finally, for Example 3 we introduce

$$
Q(\alpha, \beta, \gamma)=-\frac{d^{2}}{d v^{2}}+\alpha v^{2}+\beta v^{4}+\gamma v^{-2}
$$

and the formal eigenfunction equation, $v \geqslant 0$ :

$$
Q(\alpha, \beta, \gamma) \Psi(v ; \alpha, \beta, \gamma)=\Lambda(\alpha, \beta, \gamma) \Psi(v ; \alpha, \beta, \gamma)
$$

We can give the first set of transmutation formulae by noting:

Proposition 1.1. Equation (1.16) is equivalent to equation (1.18) under the change of variables

$$
\begin{aligned}
& \alpha=-4 \lambda ; \quad \beta=-4 f ; \quad \gamma=4 g+3 / 4 ; \quad \Lambda=4 z \\
& x=v^{2} ; \quad J(x)=v^{1 / 2} \Psi(v) .
\end{aligned}
$$

This proposition is intended in the sense of equivalence of ordinary differential equations, and not as a statement of operator equivalence. Notice that $\gamma=4 g+3 / 4$ is equivalent to $\gamma=4 m^{2}-1 / 4$. In particular, $\gamma=0$ corresponds to $g=-3 / 16(m=+1 / 4)$ and eigenfunctions of the anharmonic oscillator $-d^{2} / d v^{2}+v^{2}+\beta v^{4}$ on all of $(-\infty, \infty)$, that are even (resp. odd) under $v \rightarrow-v$ correspond to $h(-3 / 16, \Lambda / 4,-\beta / 4)+1 / 4$ with Neumann-like (resp. Dirichletlike) boundary conditions. Also, the two-dimensional oscillator $-\Delta+x^{2}+\beta x^{4}$ restricted to functions of the form $x^{1 / 2} f(x) e^{i m \phi}$, i.e.,

$$
-\frac{d^{2}}{d x^{2}}+x^{2}+\beta x^{4}+x^{-2}\left(m^{2}-1 / 4\right)
$$

corresponds to $h(g, \Lambda / 4,-\beta / 4)+1 / 4$ with $g=m^{2}-1 / 4$, so that $m$ is given by (1.8).

The second set of transformation formulae relate (1.15) when separated in parabolic coordinates to (1.18).

Proposition 1.2. If $\mathbf{r}=\left(u v \cos \phi, u v \sin \phi, \frac{1}{2}\left(u^{2}-v^{2}\right)\right) \quad($ i.e., $u=\sqrt{r+z}$, $v=\sqrt{r-z}$, so $u^{2}, v^{2}$ are parabolic coordinates), then

$$
\Theta(r)=(u v)^{-1 / 2} \chi(u) \omega(v) e^{i m \phi}
$$


obeys (1.1) if $\chi, \omega$ obey

$$
\begin{gathered}
Q\left(-E, F / 2, m^{2}-1 / 4\right) \chi=\Lambda_{1} \chi \\
Q\left(-E,-F / 2, m^{2}-1 / 4\right) \omega=\Lambda_{2} \omega \\
\Lambda_{1}+\Lambda_{2}-2 Z=0 .
\end{gathered}
$$

This is a straightforward, although tedious, change of variables; see [54; $\S 15.16$ ]. For $m \geqslant 1, Q$ is essentially self-adjoint without any boundary condition, and for $m=0$ the Dirichlet conditions correspond to the requirement that $\Theta$ be bounded.

(1.20c) is just the formal equation relating the $\Lambda$ of $(1.18)$ and the $E(Z, F)$ of (1.15):

$$
\Lambda\left(-E, F / 2, m^{2}-1 / 4\right)+\Lambda\left(-E,-F / 2, m^{2}-1 / 4\right)=2 Z .
$$

In particular, it relates the Oppenheimer formula for $E(Z, F)$ when $m=0$ and the analogue of the Bender-Wu formula for $\Lambda(\alpha,-\mu+i O,-1 / 4)$ found by Bender, $\mathrm{Wu}$, and Banks [7]. It can be proven [21] that any solution of the partial differential equation with suitable boundary conditions at infinity is a sum of products in parabolic coordinates.

As a final introductory point we should say a little about the physical interpretation of the quantity $E(F)$ as the energy of a resonance. That is, to what extent is $E(F)$ dependent on our decision to work in a framework that gives a special meaning to the dilation analytic vectors (see Simon [60] for additional discussion of this point)? First, there is a remark of Howland [61] that, carried over to our situation, gives a natural mathematical meaning to $E(F)$ independent of any special choice: namely, our results show for suitable complex $F$ (indeed for $\mathrm{F}$ purely imaginary), $E(F)$ is actually an eigenvalue of $H(F)$; thus $E(F)$ is just the analytic continuation in $F$ of an eigenvalue. From a physical point of view, one should prove that resonance energies as defined within the framework of the dilation analytic theory are the poles of the scattering operator analytically continued. For results of this genre in the case of zero field see [60]; see Yajima [57] for the case with electric field. While these results tend to suggest strongly that the $E(F)$ will be the poles of the $S$-operator for our actual case, all of them are restricted to potentials with stronger falloff than $r^{-1}$ at infinity and some require more local regularity. We also note that while there is some controversy in the physics literature about whether many body effects might produce poles of $S$ unrelated to the dilation analytic theory, there is general agreement that these will yield poles of $S$ and that in the two body case considered here, these are the only such poles. Finally, we note that Herbst [62] has related the $E(F)$ to the decay of $\left(\phi, e^{i t H(F)} \phi\right)$ for $\phi$ an eigenvector of $H(O)$; this relates another notion of "resonance" discussed in the physics literature. 
We can now describe the contents of the rest of the paper. The above transmutation formula reduces the Stark problem to an ODE, and the other two problems are already ODE's. We will characterize resonance solutions as solutions with a certain behavior in a certain direction of the complex plane. This characterization, known in the physics literature as Siegert-Gamow boundary conditions (after $[44,16]$ ), is equivalent to the characterization by dilation analyticity in the Stark case and to the characterization by analytic continuation in the anharmonic case. We will then be able to isolate $\operatorname{Im} \Lambda$ by variation of parameters starting from an approximate solution which is WKB in suitable regions, Airy at turning points and Whittaker in the near region. The basic strategy is described in Section 2. As the reader will have noticed, it is unfortunately limited to problems given by ODE's and involves some manipulation with special functions. The application to the Titchmarsh problem is complicated by the long range of the $r^{-1}$ potential. Since it is not $L^{1}$ at infinity, one cannot approximate the solution of (1.16) by solutions of Airy's equation $h(g=0, z=0, f) J=\lambda J$. The analogue of Titchmarsh's problem where $Z / r$ is replaced by a potential in $L^{1}$ is thus technically much easier, so we begin by analyzing that problem in $\$ 3$, thereby illustrating the basic strategy. The Titchmarsh problem is then analyzed in $\$ 4$. Given the analysis and the transmutation formulae it is easy to obtain the major results of this paper; a proof of the Bender-Wu formula $(1.9 / 1.11)$ in $\$ 5$ and of the Oppenheimer formula (1.5) in $\S 6$. We have included a glossary of information we need on special functions in one appendix and placed several calculations in other appendices.

It is a pleasure to thank L. Benassi, S. Graffi, and V. Grecchi for valuable correspondence, and I. Herbst for valuable discussions.

§2. The basic strategy. In this section, we want to describe the basic approach and reduce the analysis of resonance widths to the proofs of estimates on solutions of ordinary differential equations with prescribed behavior at infinity. We begin by relating resonances to these special solutions, thereby getting an implicit equation. By analyzing this equation with Taylor's Theorem, we will obtain the required reduction. Finally we will give several general elements of the construction of solutions and their estimation.

Definition. By $\mathfrak{V}$ we denote the class of functions $V(x)$ on $(0, \infty)$ which have an analytic continuation to the region $S=\{z|| \arg z \mid<\pi / 3+\delta\}$ for some $\delta>0$ and which obey either

$$
\int^{\infty}\left|V\left(a+x e^{i \phi}\right)\right| d x<\infty
$$

for all $a \in S,|\phi|<\pi / 3+\delta$, or

$$
V=V_{1}+V_{2}+V_{3},
$$


where $V_{1}=Z / x, V_{2}=\gamma / x^{2}, \gamma \geqslant-1 / 4$, and $V_{3}$ obeys (2.1). Note that we do not demand that $V$ be real. This will be important because the transmutation formulae lead to nonreal $V$ 's.

Proposition 2.1. Let $V \in \mathfrak{V}$; then for every $F>0$ and $E \in \mathrm{C}$, the differential equation

$$
-\psi^{\prime \prime}-(F x-V(x)) \psi=E \psi
$$

has a solution $\psi=J(x, F, E)$ that, together with its derivative, goes to zero in the sector $0<\arg x<\pi / 3+\delta$, uniformly in the regions $\epsilon<\arg x<\pi / 3+\delta-\epsilon$. All such solutions of (2.2) are multiples of $J$. This solution satisfies $|J| \leqslant$ $C \exp \left(-\epsilon|z|^{3 / 2}\right)$ in $\{z|| z \mid>R, \arg Z \in(\delta, \pi / 3-\delta)\}$, while all other solutions go to infinity.

Proof. Uniqueness follows from the constancy of the Wronskian of two solutions. Existence follows from a simple variation of parameters of the type we consider extensively below with either Airy functions (the exact solutions when $V=0$ ) or with the WKB solutions. We will remark on some details of this construction below.

Definition. By a resonance solution of the operator $-d^{2} / d x^{2}+V-F x$ with prescribed boundary condition $C$ at $x=0$, we mean a solution $J$ of (2.2) with the vanishing conditions given in Proposition 2.1, which moreover obeys the prescribed condition $C$. The corresponding value of $E$ is called a resonance energy.

We must link this definition to the operator-theoretic definitions alluded to in $\S 1$, namely those given by dilation analyticity in the Stark and Titchmarsh problems and by analytic continuation in a parameter in the anharmonic oscillator.

Proposition 2.2. In the following cases:

(a) $V$ regular at $x=0 ;-d^{2} / d x^{2}$ with Dirichlet or Neumann conditions,

(b) $V \sim Z / x$ at $x=0,-d^{2} / d x^{2}$ with Dirichlet conditions, or

(c) $V \sim Z / x+l(l+1) / x^{2}(l \geqslant 1)$ at $x=0,-d^{2} / d x^{2}+V$ with the boundary conditions (Dirichlet) obtained by closing the operator on $C^{\infty}(0, \infty)$,

Herbst's method [24] of dilation analyticity is applicable, and resonance in the Herbst theory corresponds precisely to resonance energy as defined above.

Proof. Herbst's theory depends on showing that $-e^{-2 i \theta} d^{2} / d x^{2}+V\left(e^{i \theta} x\right)-$ $F e^{i \theta} x$ has discrete spectrum for $\theta$ real, small and non-zero. Resonances are eigenvalues for $\operatorname{Im} \theta$ small and positive and are independent of $\theta$. It is easy to see that resonances thus correspond to solutions of the ODE which are squareintegrable along rays $\left\{x e^{i \theta} \mid x>0, \theta\right.$ fixed, small, and positive $\}$ and hence to resonances in our sense.

Proposition 2.3. Fix $\alpha, \gamma$ real, $\gamma \geqslant-1 / 4, \alpha \geqslant 0$. Let $\Lambda(\beta)$ be defined for $\beta>0$ as an eigenvalue of the operator $Q$ of (1.17) and define $\Lambda(\beta)$ for $\arg \beta=i \pi$ 
by analytic continuation in the lower half plane. Then $\Lambda(-|\beta|-i O)$ is such a continued eigenvalue, if and only if $-1 / 4 \alpha$ is a resonance eigenvalue for the Hamiltonian (1.7) with $g=\frac{1}{4}(\gamma-3 / 4), z=\frac{1}{4} \Lambda f=-\frac{1}{4} \beta=\frac{1}{4}|\beta|$ and with the appropriate conditions.

Remarks. 1. By the appropriate boundary conditions, we mean that Dirichlet-like (resp. Neumann-like) boundary conditions in (1.17) correspond to the same type of condition in (1.7).

2. We note that in the $\gamma=0$ problem the whole line reduces by symmetry to the direct sum of problems restricted to even and odd functions, and these are equivalent to the Neumann and Dirichlet problems on the half-line.

Proof. One first notes that if $\beta$ is held fixed and positive and $\gamma$ is fixed, $\gamma \geqslant-1 / 4$, then analytic continuation in $\alpha$ is possible on an operator theory level for all $\Lambda, \alpha[48]$, and eigenvalues correspond to solutions which go to zero at infinity along the real axis. If one notes that under the scaling $x \rightarrow \lambda x$ the differential operator $Q(\alpha, \beta, \gamma)$ goes to $\lambda^{-2} Q\left(\alpha \lambda^{4}, \beta \lambda^{6}, \gamma\right)$ one can relate solutions of $Q(x, \beta, \gamma) \psi=\Lambda \psi$ going to zero as $|x| \rightarrow \infty, \arg x=0$ to solutions of $\lambda^{-2} Q\left(\alpha \lambda^{4}, \beta \lambda^{6}, \gamma\right)=\Lambda \eta$ going to zero as $|x| \rightarrow \infty, \arg (\lambda x)=0$. This continuation in $\beta$ corresponds to continuation in $\alpha$ if one remembers to continue the $\arg x$ for which the boundary condition is demanded. This proves the result. To see that continuation of $\beta$ in the lower half plane is involved note that the condition is $\frac{1}{6} \arg \beta+\arg x=0$.

Proposition 2.4. Fix $m=0,1 \ldots$ and let $\Lambda\left(\alpha, \beta, m^{2}-1 / 4\right)$ be the real eigenvalues of $Q\left(\alpha, \beta, m^{2}-1 / 4\right)$ for $\beta>0$ and the resonance eigenvalues of $\beta<0$. Then for fixed $Z, F$ real and positive, solutions $E$ of $\left(1.20 c^{\prime}\right)$ correspond to resonances in Herbst's sense for the Stark problem (1.15).

Proof. This involves the same argument as in Proposition 2.2 and the parabolic separation ' variables.

Remarks. 1. It can be proven [21] that every resonance wave function for the Stark problem is a product in parabolic coordinates, so that every such resonance corresponds to a solution of $\left(1.20 \mathrm{c}^{\prime}\right)$.

2. In terms of analytic continuation in $\beta$, one often writes $\left(1.20 c^{\prime}\right)$ with $-F / 2=e^{-i \pi} F / 2$ to indicate the continuation through the lower half-plane.

These considerations reduce the study of resonances in the operator-theoretic sense to the finding of what we have called resonance solutions, and henceforth we only consider these resonance solutions.

In addition to the time-independent definitions of resonances we have mentioned, there is a time-dependent point of view, from which a resonance state is a localized state which, while not an eigenfunction, decays (in time) abnormally slowly. The shortcoming of this point of view is its imprecision. By looking at spectral projections onto small enough intervals one can find arbitrarily slowly decaying states, but the slower the decay the worse the 
localization. There is no convincing canonical balance of localization and slowness of decay to optimize. However, a representative time-dependent resonance state can be constructed from the resonance solution we write down by cutting it off so that it becomes $L^{2}$, and the rate of decay is essentially the imaginary part of the resonance energy that we calculate by a golden-rule argument (see Section 3).

The procedure we will follow yields resonances in the Stark problem as solutions of an implicit equation. Our strategy below will be to solve the other problems also in the form of implicit equations. Thus we will want to solve an equation

$$
G(f, \lambda)=0
$$

for $\lambda$ as a function of $f$, related to a real solution $\lambda_{0}$ of $G\left(0, \lambda_{0}\right)=0$. Under suitable conditions on $G$ we know that for $f$ small there is a unique solution of (2.3) with $\lambda(f)$ near $\lambda_{0}$. We begin with the rather elementary

THEOREM 2.5. Let $G(f, \lambda)$ be a $C^{1}$ function on $\left\{f|f \in[0, F),| \lambda-\lambda_{0} \mid<\epsilon, \lambda\right.$ complex $\}$ which is analytic in $\lambda$ for each fixed $f$ and with

(a) $G\left(0, \lambda_{0}\right)=0$ and $\partial G(0,0) / \partial \lambda$ is a nonzero real number; and

(b) for some $\alpha \geqslant 0$ :

$$
\frac{\partial G}{\partial \lambda}(f, \lambda)-\frac{\partial G}{\partial \lambda}\left(0, \lambda_{0}\right)=O\left(\left|\lambda-\lambda_{0}\right|^{\alpha}+|f|^{\alpha}\right)
$$

(c) for a fixed function $g(f)$ and for $\lambda$ real and near $\lambda_{0}$ :

$$
\operatorname{Im} G(f, \lambda)=g(f) h(\lambda) \exp (-a(\lambda) / f)\left(1+O\left(|f|^{a}\right)\right)
$$

for a $C^{1}$ function $a$ of $\mu$ with $h$ smooth and $h\left(\lambda_{0}\right) \neq 0$. Then there is a unique solution $\lambda(f)$ near $\lambda_{0}$ of $G(f, \lambda(f))=0$ for $f$ small. Moreover, $\operatorname{Re} \lambda(f)=\lambda_{0}+\lambda_{1} f+$ $o(f)$ for some $\lambda_{1 j}$ and

$$
\operatorname{Im} \lambda(f)=-C g(f) h\left(\lambda_{0}\right) \exp \left(-a\left(\lambda_{0}\right) / f\right) \cdot(1+o(1)),
$$

where

$$
C=\left(\frac{\partial G}{\partial \lambda}\left(0, \lambda_{0}\right)\right)^{-1} \exp \left(-\lambda_{1} \frac{d a}{d \mu}\left(\mu=\lambda_{0}\right)\right)
$$

If the $o(f)$ term in $\operatorname{Re} \lambda(f)$ can be replaced by $O\left(|f|^{1+a}\right)$, then the $o(1)$ term in $\operatorname{Im} \lambda(f)$ can be replaced by $O\left(|f|^{a}\right)$.

Remark. By $C^{1}$ on the closed interval, we mean in the sense of a one-sided derivative.

Proof. By the implicit function theorem, there is a unique $\lambda(f)$ which is $C^{1}$ so that $\lambda(f)=\lambda_{0}+\lambda_{1} f+o(f)$. Using the mean-value theorem, we find $\mu(f)$ with 
$\operatorname{Re} \mu(f)=\operatorname{Re} \lambda(f)$ and $\operatorname{Im} \mu(f)$ between 0 and $\operatorname{Im} \lambda(f)$ so that

$$
\operatorname{Im} G(f, \operatorname{Re} \lambda(f))=-\operatorname{Im} \lambda \operatorname{Re} \frac{\partial}{\partial \lambda} G(f, \mu(f)),
$$

where we have used $\operatorname{Im} G\left(f_{o}, \lambda(f)\right)=0$. Since

$$
\exp (-a(\operatorname{Re} \lambda(f)) / f)=\exp \left(-a\left(\lambda_{0}\right) / f\right) \cdot \exp \left(-\operatorname{Re} \lambda_{1}(d a / d \mu)+o(1)\right),
$$

the result follows.

Under suitable hypotheses on $G$, one can go to higher order in $\operatorname{Im} \lambda(f)$, in the sense that $(1+o(1))$ can be replaced by $\left[1+a f+b f^{2}+o\left(f^{2}\right)\right]$ for some explicit $a, b$. In particular, one could use the methods of this paper to go to higher order in the Oppenheimer and Bender-Wu formulae.

The final element of the overall strategy involves the implicit equation we will use to find the eigenvalues. This will be the standard method [40,22] of taking the Wronskian of two solutions of the basic differential equation, one obeying the boundary condition at infinity, the other at the origin. To construct the solution of an equation

$$
\left(-\frac{d^{2}}{d x^{2}}+C(x)\right) \psi(x)=0
$$

with some boundary conditions at $x_{0}$, we use as comparison functions two functions $\phi_{ \pm}$obeying

$$
\left(-\frac{d^{2}}{d x^{2}}+A(x)\right) \phi_{ \pm}(x)=0
$$

If we expand

$$
\begin{aligned}
\psi(x) & =a_{+}(x) \phi_{+}(x)+a_{-}(x) \phi_{-}(x) \\
\psi^{\prime}(x) & =a_{+}(x) \phi_{+}^{\prime}(x)+a_{-}(x) \phi_{-}^{\prime}(x),
\end{aligned}
$$

then we obtain the equivalent equation

$$
\frac{d}{d x}\left(\begin{array}{l}
a_{+} \\
a_{-}
\end{array}\right)=\frac{B(x)}{W\left\{\phi_{-}, \phi_{+}\right\}}\left[\begin{array}{cc}
\phi_{+} \phi_{-} & \phi_{-}^{2} \\
-\phi_{+}^{2} & -\phi_{+} \phi_{-}
\end{array}\right]\left(\begin{array}{l}
a_{+} \\
a_{-}
\end{array}\right),
$$

where $B(x)=C(x)-A(x)$ and $W\{f, g\} \equiv f g^{\prime}-g f^{\prime}$ is the Wronskian of $f$ and $g$. This use of variation of parameters to justify WKB-type results has been used before by various authors $[15,22,11]$. In all cases we construct resonance solutions of (2.2) when $E$ is real and negative by performing the variation of parameters along a path $P=P_{1} \cup P_{2}$ :

$$
\begin{aligned}
& P_{1}=\{x \mid 0<x<-E / F\} \\
& P_{2}=\left\{x \mid x=-E / F+r e^{i \pi / 3}, r>0\right\} .
\end{aligned}
$$


In the region

$$
P_{1}^{(0)}=\left\{x \in P_{1} \mid x<F^{-\alpha}\right\}
$$

(where $\alpha$ is a fixed number strictly between 0 and $1 / 2$ to be picked for convenience later), we will take $\phi_{ \pm}$to be solutions of (2.2) with $F=0$; and in the regions $P_{2}$ and

$$
P_{1}^{(1)}=\left\{x \in P_{1} \mid x<F^{-\alpha}\right\}
$$

we would like to take $\phi_{ \pm}$to be solutions of (2.2) with $V=0$. We can get away with this in the short-range case (\$3), and only need to consider three regions, $P_{1}^{(0)}, P_{1}^{(1)}$, and $P_{2}$. In the long-range case $\left(V \sim x^{-1}\right)$, however, we cannot get away with using solutions of (2.2) with $V=0$, because in the limit $F=0$ the solutions of $(2.2)$ are not asymptotically $\exp ( \pm \sqrt{-E} x)$, but rather $\exp ( \pm \sqrt{-E}(x+c \ln x))$. We therefore need to take $\phi_{ \pm}$to be WKR functions in order to get the right asymptotics. Since such approximations are poor near the classical turning point $-E / F$, the long-range case (\$4) will need to break $P_{1}^{(1)}$ into two regions

$$
\begin{aligned}
& P_{1}^{(2)}=\left\{x \in P_{1} \mid F^{-\alpha} \leqslant x \leqslant[-E / F]-F^{-\beta}\right\} \\
& P_{1}^{(3)}=\left\{x \in P_{1} \mid[-E / F]-F^{-\beta} \leqslant x\right\} .
\end{aligned}
$$

Likewise, $P_{2}$ must be broken into two regions,

$$
\begin{aligned}
& P_{2}^{(1)}=\left\{x \in P_{2}|r=| x+E / F \mid \leqslant F^{-\beta}\right\} \\
& P_{2}^{(2)}=\left\{x \in P_{2} \mid r \geqslant F^{-\beta}\right\} .
\end{aligned}
$$

In the region $P_{1}^{(1)}$ we will take solutions with $F=0$ (Whittaker functions); in the regions $P_{1}^{(2)}$ and $P_{2}^{(2)}$, we will use WKB approximations; and in the regions $P_{1}^{(3)}$ and $P_{2}^{(1)}$ (near the turning point) we will take Airy functions in conformity with the ideas of Langer [32].

One further complication is that it will be convenient not to have $\phi_{ \pm} \in C^{1}$ at the matching point for regions $P_{1}$ and $P_{2}$-this is no problem since only $\psi$ and $\psi^{\prime}$ need be continuous. For this reason we will give different names (to wit $\phi_{ \pm}$and $\eta_{ \pm}$) to the bases in the two regions. We will rely on a general property of solutions of (2.6), which is the remarkable fact that when $A, C$, and $\phi_{ \pm}$are real valued, $\operatorname{Im}\left(a_{+} / a_{-}\right)$varies only slowly, and according to an explicit formula:

TheOREM (2.6). Let $\phi_{ \pm}$and $B$ be as above and real-valued on the real interval $\left[x_{0}, x_{1}\right]$, and let $a_{ \pm}$obey (2.6). Then

$$
\operatorname{Im}\left(\frac{a_{+}}{a_{-}}\right)\left(x_{0}\right)=\operatorname{Im}\left(\frac{a_{+}}{a_{-}}\right)\left(x_{1}\right) \exp (X),
$$


where

$$
X=-\int_{x_{0}}^{x_{1}} 2 B(x)\left[W\left\{\phi_{-}, \phi_{+}\right\}\right]^{-1}\left[\phi_{+}(x) \phi_{-}(x)+\phi_{+}^{2}(x) \operatorname{Re}\left(\frac{a_{+}}{a_{-}}\right)(x)\right] d x .
$$

Proof. Since

$$
\left(\frac{a_{+}}{a_{-}}\right)^{\prime}=\frac{a_{+}^{\prime}}{a_{-}}-\left(\frac{a_{+}}{a_{-}}\right)\left(\frac{a_{-}^{\prime}}{a_{-}}\right)
$$

the differential equation leads to:

$$
\left(\frac{a_{+}}{a_{-}}\right)^{\prime}=\frac{B(x)}{W\left\{\phi_{-}, \phi_{+}\right\}}\left[2 \phi_{+} \phi_{-}\left(\frac{a_{+}}{a_{-}}\right)+\phi_{-}^{2}+\phi_{+}^{2}\left(\frac{a_{+}}{a_{-}}\right)^{2}\right] .
$$

Taking the imaginary part of both sides,

$$
\frac{d}{d x} \ln \left[\operatorname{Im}\left(\frac{a_{+}}{a_{-}}\right)\right]=\frac{2 B(x)}{W\left\{\phi_{-}, \phi_{+}\right\}}\left[\phi_{+} \phi_{-}+\phi_{+}^{2} \operatorname{Re}\left(\frac{a_{+}}{a_{-}}\right)\right],
$$

and integrating this leads to (2.10), (2.11).

We emphasize that the errors introduced in (2.10) when $X$ is small and $\exp (X)$ is expanded in $X$ are multiplicative, i.e.,

$$
\operatorname{Im}\left(\frac{a_{+}}{a_{-}}\right)\left(x_{0}\right)=\operatorname{Im}\left(\frac{a_{+}}{a_{-}}\right)\left(x_{1}\right) \cdot[1+O(X)] .
$$

§3. The short-range case: A warm-up. As we explained at the end of the last section, the treatment of the Titchmarsh problem is complicated by the long range nature of $x^{-1}$. In addition, there are complications due to the singularities of $x^{-1}$ and $x^{-2}$ at $x=0$. Thus we illustrate the situation by considering

$$
H_{0}=\frac{-d^{2}}{d x^{2}}+V(x)
$$

on $L^{2}(0, \infty)$ with the boundary condition

$$
u(x=0)=0
$$

We let

$$
H_{f}=H_{0}-f x
$$


and consider a negative eigenvalue $\lambda_{0}$ of $H_{0}$. We suppose throughout that $V(x)$ is analytic in

$$
\begin{gathered}
\left\{x|| \arg x \mid<\frac{\pi}{3}+\delta\right\} \equiv R_{\delta} \\
|V(x)| \leqslant C(1+|x|)^{-1-\epsilon}
\end{gathered}
$$

for some $\epsilon, \delta$ positive, and

$$
V(x) \text { is analytic near } x=0 .
$$

Under the hypotheses (3.4), we can define four solutions $\sigma_{ \pm}, \sigma_{D}, \sigma_{N}$ of

$$
-\sigma^{\prime \prime}(x)+V(x) \sigma(x)=\lambda \sigma(x) .
$$

The solutions $\sigma_{D, N}(x, \lambda)$ obey the boundary conditions

$$
\sigma_{D}(0, \lambda)=0, \quad \sigma_{D}^{\prime}(0, \lambda)=1 ; \quad \sigma_{N}(0, \lambda)=1, \quad \sigma_{N}^{\prime}(0, \lambda)=0
$$

One easily sees that $\sigma_{D, N}(x, \lambda)$ are analytic in $R_{\delta} \times \mathrm{C}$ and obey:

$$
\left|\sigma_{D, N}(x, \lambda)\right|+\left|\sigma_{D, N}^{\prime}(x, \lambda)\right| \leqslant C \exp \left(|\lambda|^{1 / 2}|x|\right)
$$

in the region $R_{\delta / 2} \times \mathrm{C}$. The solution $\sigma_{-}$is defined by the conditions:

$$
\begin{gathered}
\sigma_{-}(x, \lambda) / \exp (-\sqrt{-\lambda} x) \rightarrow 1 \\
\sigma_{-}^{\prime}(x, \lambda) /[\exp (-\sqrt{-\lambda} x)](-\sqrt{-\lambda}) \rightarrow 1
\end{gathered}
$$

as $x \rightarrow \infty$ along the real axis. $\sigma_{-}$is analytic in $R_{\delta} \times\{\lambda \mid \lambda \in[0, \infty)\}$ and is easily constructed with a variation of parameters about $\exp ( \pm \sqrt{-\lambda} x)$; see, e.g., Dicke (appendix to [48]). The solution $\sigma_{+}$is to be chosen so that

$$
W\left\{\sigma_{-}, \sigma_{+}\right\}=2 \sqrt{-\lambda} .
$$

For example, we can take

$$
\sigma_{+}=\sigma_{N}+\left[\sigma_{-}^{\prime}(0)\right]^{-1}\left[\sigma_{-}^{\prime}(0)-2 \sqrt{-\lambda}\right] \sigma_{D}
$$

which is meromorphic in $R_{\delta} \times\{\lambda \mid \lambda \in(0, \infty)\}$ with poles in $\lambda$ only at points with $\sigma_{-}^{\prime}(0, \lambda)=0$. It is not hard to see that $\sigma_{+}$obeys:

$$
\begin{gathered}
\sigma_{+}(x, \lambda) / \exp (+\sqrt{-\lambda} x) \rightarrow 1, \\
\sigma_{+}^{\prime}(x, \lambda) / \exp (+\sqrt{-\lambda} x)(\sqrt{-\lambda}) \rightarrow 1 .
\end{gathered}
$$


Equation (3.8) determines $\sigma_{+}$up to multiples of $\sigma_{-}$, and, in particular, if

$$
\sigma_{-}\left(0, \lambda_{0}\right)=0
$$

then $\sigma_{+}\left(0, \lambda_{0}\right)$ is independent of any choice. We will prove the following result in this section:

Theorem 3.1. Assume (3.4) holds, and let $\lambda_{0}$ be a negative eigenvalue of $H_{0}$ (so that (3.9) holds). Then $H_{f}$ has a resonance $\lambda(f)$ near $\lambda_{0}$ for $f$ small and real, and for some $a>0$,

$$
\operatorname{Im} \lambda(f)=C \exp \left(-4\left(-\lambda_{0}\right)^{3 / 2} / 3 f\right) \cdot\left[1+O\left(f^{a}\right)\right]
$$

where

$$
C=(1 / 8) \exp (\gamma)\left(-\lambda_{0}\right)^{-1 / 2} \sigma_{+}\left(0, \lambda_{0}\right)\left[\frac{\partial}{\partial \lambda}\left(\sigma_{-}(0, \lambda) / \sqrt{-\lambda}\right)\right]^{-1}
$$

and

$$
\gamma=\frac{-2\left(-\lambda_{0}\right)^{1 / 2} \int_{0}^{\infty} x\left[\sigma_{-}\left(x ; \lambda_{0}\right)\right]^{2} d x}{\int_{0}^{\infty}\left[\sigma_{-}\left(x, \lambda_{0}\right)\right]^{2} d x}
$$

Remark. The $\exp (\gamma)$ term is somewhat subtle; it arises from the $\lambda_{1}(d a / d \mu)$ term in Theorem 2.5. In physicist's language, it comes from the effect that the first-order energy shifts have on the tunneling. This is obviously a negligible effect for the anharmonic oscillator, which is reflected in the transformation formula in that if one adjusts the charge rather than the energy to accommodate the change in $f$, then there is no such term. In the Stark-effect problem, no such term occurs for the ground state, for which $E-E_{0}=O\left(F^{2}\right)$, but one is present for certain excited states; see $\$ 6$.

To prove Theorem 3.1, we construct a solution $J(x, f, \lambda)$ of (2.2) with $\lambda=E$ by a variation of parameters using solutions $\phi_{ \pm}(x, f, \lambda)$ such that $J(x, f, \lambda)=$ $a_{+}(x, f, \lambda) \phi_{+}(x, f, \lambda)+a_{-}(x, f, \lambda) \phi_{-}(x, f, \lambda) . J$ will be chosen to go to zero as $|x| \rightarrow \infty$ with $0<\arg x<\pi / 3$, so that $\lambda(f)$ is the solution of

$$
J(x=0, f, \lambda)=0 .
$$

It will be convenient to replace $(3.11)$ by the equivalent condition

$$
\begin{aligned}
& G(f, \lambda)=0 \\
& G(f, \lambda)=\phi_{-}(0)+\phi_{+}(0)\left(\frac{a_{+}}{a_{-}}\right)(x=0)=\frac{J(x=0)}{a_{-}(x=0)} .
\end{aligned}
$$

The hypotheses (a), (b) of Theorem 2.5 are easy to check in the construction below (done for $\lambda$ complex), so we will concentrate on verifying hypothesis (c) 
and identifying $\operatorname{Im} G(f, \mu)$. Since (c) involves only real $\lambda$, we will henceforth take $\lambda$ real.

We will choose $\phi_{ \pm}$in such a way that for $f=0, a_{+} / a_{-}(x=0)=0$ independently of $\lambda$, and thus if we verify the hypothesis of Theorem 2.5:

$$
\operatorname{Im} \lambda(f)=-\exp (\gamma) \phi_{+}(0)\left[\frac{\partial \phi_{-}}{\partial \lambda}(0) \operatorname{Im}\left(\frac{a_{+}}{a_{-}}\right)\right](x=0) \cdot\left(1+O\left(f^{a}\right)\right),
$$

where $\phi_{ \pm}(0)$ means $\phi_{ \pm}$evaluated at $\lambda=\lambda_{0}, f=0, x=0$. Moreover, at $f=0, \phi_{ \pm}$ will be chosen to be multiples of $\sigma_{ \pm}$, so that $\operatorname{Im} \lambda(f)$ will depend on the calculation of $\operatorname{Im}\left(a_{+} / a_{-}\right)(x=0)$, and hence Theorem 2.6 will be useful.

As described in Section 2, we define $\phi_{ \pm}$to be $C^{1}$ on $[0,-\lambda / f]$ solving (2.5) with

$$
A(x, \lambda)= \begin{cases}V(x)-\lambda, & x<f^{-\alpha} \\ -f x-\lambda, & x>f^{-\alpha}\end{cases}
$$

and with $\alpha$ fixed once and for all, $0<\alpha<1 / 2$. Thus $a_{ \pm}$will obey (2.6) with

$$
B(x, \lambda)= \begin{cases}-f x, & x<f^{-\alpha} \\ V(x), & x>f^{-\alpha}\end{cases}
$$

$\phi_{ \pm}$can be expanded in terms of the basic solutions $\sigma_{ \pm}$in the region $x<f^{-\alpha}$, and in the region $x>f^{-\alpha}$ we use the functions

$$
\begin{aligned}
& a(x ; f, \lambda)=A i\left(-f^{1 / 3}(x+\lambda / f)\right) \\
& b(x ; f, \lambda)=B i\left(-f^{1 / 3}(x+\lambda / f)\right)
\end{aligned}
$$

where $A i, B i$ are Airy functions (see Appendix b); Bi is chosen so that $b$ falls off from $x=0$ to $x=-\lambda / f$. $\phi_{ \pm}$are determined by the differential equation and the "boundary conditions":

$$
\begin{aligned}
& \phi_{+}=\sigma_{+}(x, \lambda), \quad x \in P_{1}, x<f^{-\alpha}, \\
& \phi_{-}=N(f, \lambda) b(x, f, \lambda), x \in P_{1}, x>f^{-\alpha},
\end{aligned}
$$

where $N$ is chosen so that

$$
W\left\{\phi_{-}, \phi_{+}\right\}=1,
$$

i.e.,

$$
N(f, \lambda)=\left[W\left\{b, \sigma_{+}\right\}\left(x=f^{-\alpha}\right)\right]^{-1} .
$$

(Note: Most Wronskians we consider are of solutions of the same second-order equations, so we need not specify a point $x$. In (3.12) and in some formulae 
below we do wish to specify a point $x$; actually, as we shall see, our value of $\alpha$ is irrelevant in (3.14) to leading order in $f$.) The evaluation of $N$ and of the expansions

$$
\begin{array}{ll}
\phi_{+}(x)=C_{+}(a) a(x, f, \lambda)+C_{+}(b) b(x, f, \lambda), & x \in P_{1}, x>f^{-\alpha}, \\
\phi_{-}(x)=C_{-}\left(\sigma_{+}\right) \sigma_{+}(x, f, \lambda)+C_{-}\left(\sigma_{-}\right) \sigma_{-}(x, f, \lambda), & x \in P_{1}, x<f^{-\alpha},
\end{array}
$$

are simple exercises of the use of Wronskian equalities at $x=f^{-\alpha}$; for example,

$$
C_{+}(a)=W\left\{\sigma_{+}, b\right\}\left(x=f^{-\alpha}\right) / W\{a, b\} .
$$

As $f \rightarrow 0$, the point $x=f^{-\alpha}$ is in the region where $\sigma_{ \pm}, \sigma_{ \pm}^{\prime}$ obey their asymptotic formulae (3.7) and where $a, a^{\prime}, b, b^{\prime}$ obey asymptotic formulae (b.9, b.10) given in Appendix B. Thus, one can obtain asymptotic formulae for the constants $C_{ \pm}$ and for $N$; explicitly:

$$
\phi_{+}(x ; f, \lambda)=C_{+}(a) a(x, f, \lambda) \cdot(1+O(f)),
$$

and

$$
C_{+}(a)=Z f^{-1 / 6} \pi^{1 / 2}(-\lambda)^{1 / 4} \exp \left(\frac{2}{3}(-\lambda)^{3 / 2} / f\right) \cdot(1+O(f))
$$

uniformly in $\left[f^{-\alpha},-\lambda / f\right]$;

$$
\phi_{-}(x, f, \lambda)=C_{-}\left(\sigma_{-}\right) \sigma_{-}(x, \lambda) \cdot(1+O(f)),
$$

and

$$
C_{-}\left(\sigma_{-}\right)=N f^{1 / 6} \pi^{-1 / 2}(-\lambda)^{-1 / 4} \exp \left(\frac{2}{3}(-\lambda)^{3 / 2} / f\right) \cdot(1+O(f))
$$

uniformly in $\left[0, f^{-\alpha}\right]$; and

$$
N=\frac{1}{2}(-\lambda)^{-1 / 4} \pi^{1 / 2} f^{-1 / 6} \exp \left(-\frac{2}{3}(-\lambda)^{3 / 2} / f\right) \cdot(1+O(f))
$$

Thus (3.15), (3.14b) imply that

$$
C_{-}\left(\sigma_{-}\right)=\frac{1}{2}(-\lambda)^{-1 / 2}(1+O(f)),
$$

which is as it should be, since $W\left\{\sigma_{-}, \sigma_{+}\right\}=2 \sqrt{-\lambda}, W\left\{\phi_{-} \phi_{+}\right\}=1, \phi_{+} \sim \sigma_{+}$, and $\phi_{-} \sim C_{-} \sigma_{-}$.

One can see why all formulae are independent of $\alpha$, and why $\phi_{+} \sim C_{+}(a) \sigma_{+}$ and $\phi_{-} \sim C_{-}\left(\sigma_{-}\right) \sigma_{-}$to leading order as follows: In the asymptotic regime, the exponentially decreasing (resp. increasing) solution must match to that solution which decreases (resp. increases), and it doesn't matter much where the matching is done, since in both cases the matching is taking place in a region where both $V(x)$ and $-f x$ are small, so that $\exp ( \pm \sqrt{-\lambda} x)$ dominates. Note that while $A i$ 
falls off exponentially, that means that as $x$ is increased, $a$ will grow exponentially!

Along the curve $P_{2}$ we will make a variation of parameters with

$$
A(x, f, \lambda)=-f x-\lambda \text {. }
$$

We will use comparison functions $\eta_{ \pm}$. Since $\phi_{-} \sim b$ does not fall off exponentially along $P_{2}$, we will not take $\eta_{-}=\phi_{-}$, but rather we use the Airy function

$$
c(x,-f, \lambda)=A i\left(-f^{1 / 3}\left(x+\lambda f^{-1}\right) e^{+2 \pi i / 3}\right)
$$

and take

$$
\eta_{-}=N e^{+\pi i / 6} \quad c(x, f, \eta)
$$

with $N$ the normalization factor determined by (3.14). $\eta_{+}$will be the analytic continuation of $\phi_{+}$, so that by (3.13):

$$
\eta_{+}(x, f, \lambda)=4 \pi f^{-1 / 3} N^{-1} a(x, f, \lambda) \cdot(1+O(f)) .
$$

Notice that

$$
\begin{aligned}
W\left\{\eta_{-}, \eta_{+}\right\} & \simeq 4 \pi f^{-1 / 3} e^{+\pi i / 6} W\{c, a\} \cdot(1+O(f)) \\
& =2 \cdot(1+O(f))
\end{aligned}
$$

by (b.8).

We can now describe our construction of the resonance solution $J$ precisely. We will expand

$$
\begin{aligned}
& J(x)=b_{+} \eta_{+}+b_{-} \eta_{-}, \\
& J^{\prime}(x)=b_{+} \eta_{+}^{\prime}+b_{-} \eta_{-}^{\prime}
\end{aligned}
$$

on $P_{2}$ and seek a solution of the differential equation with $\left(b_{+}, b_{-}\right) \rightarrow(0,1)$. Of course, we will need to show that $b_{+}$is very small, so that $b_{+} \eta_{+} / \eta_{-} \rightarrow 0$. The continuity of $J$ and $J^{\prime}$ at the matching point $x=-\lambda f^{-1}$ requires that

$$
a_{+}(x=-\lambda / f)=b_{+}(-\lambda / f)-b_{-}(\lambda / f) W\left\{\eta_{-}, \phi_{-}\right\}
$$

and

$$
a_{-}(x=-\lambda / f)=b_{-}(-\lambda / f) W\left\{\eta_{-}, \eta_{+}\right\},
$$

since $\phi_{+}=\eta_{+}$and $W\left\{\phi_{+}, \phi_{-}\right\}=-1$. In particular,

$$
\operatorname{Im}\left(\frac{a_{+}}{a_{-}}\right)(x=-\lambda / f)=\operatorname{Im} \frac{1}{W\left\{\eta_{-}, \eta_{+}\right\}} \frac{b_{+}}{b_{-}}-\operatorname{Im} \frac{W\left\{\eta, \phi_{+}\right\}}{W\left\{\eta, \eta_{+}\right\}} .
$$


Now:

$$
W\left\{\eta_{-}, \phi_{-}\right\}=N^{2} e^{\pi i / 6} W\{c, b\}=\frac{N}{2 \pi} f^{1 / 3} i \equiv T i .
$$

Theorem 3.1 will follow from two propositions:

Proposition 3.2. There is a solution $J$ of $H_{f} J=\lambda J$ along the curve $P_{2}$ with

$$
b_{-}=1+O\left(|f|^{a}\right)
$$

uniformly in $x \in P_{2}$ for some $a>0$, and

$$
b_{+}=O\left(\eta_{-}^{2}\right) O\left(|f|^{a}\right)
$$

uniformly in $x$ and $f$. Moreover,

$$
b_{+}(-\lambda / f)=O\left(|f|^{a}\right) \times T,
$$

where $T$ is the quantity defined in (3.20b).

Proposition 3.3. The solution $J$ of Proposition 3.2, continued along $P_{1}$ and expanded in terms of $\phi_{ \pm}$obeys

$$
\begin{aligned}
& a_{-}(x)=2+O\left(|f|^{a}\right) \\
& a_{+}(x)=O\left(|f|^{a}\right) g(x),
\end{aligned}
$$

where

$$
g(x)= \begin{cases}1, & x<R_{0} \\ \phi_{-}^{2}(x), & x \geqslant R_{0}\end{cases}
$$

for some fixed $R_{0}$.

Proof of Theorem 3.1 (assuming Propositions 3.1 and 3.3). We first claim that the solution $J$ of Proposition 3.2 obeys resonance boundary conditions at infinity. As noted after (3.18) this requires that $b_{+} \eta_{+} / \eta_{-} \rightarrow 0$ as $x \rightarrow \infty$. But by (3.21b), for $f$ fixed, $b_{+} \eta_{+} / \eta_{-}=O\left(\eta_{+} \eta_{-}\right)=O(a c)=O\left(|x|^{-1 / 2}\right)$ by formulae (b.11), (b.12) in Appendix B. Therefore the resonance condition is given by (3.11), (3.12), if

$$
\phi_{-}(x=0)+\phi_{+}(x=0)\left(\frac{a_{+}}{a_{-}}\right)(x=0)=G(f, \lambda)=0 .
$$

Next, we claim that

$$
\operatorname{Im} \frac{a_{+}}{a_{-}}(x=0)=\operatorname{Im}\left(\frac{a_{+}}{a_{-}}\right)(x=-\lambda / f) \cdot\left(1+O\left(|f|^{a}\right)\right) .
$$

By Theorem (2.6), this follows from showing that the quantity $X$ of (2.11) is 
$O\left(|f|^{a}\right)$ with $x_{0}=0, x_{1}=-\lambda / f$. By the formula (3.22) for the asymptotics of $a_{ \pm}, \phi_{+}(x) \phi_{-}(x)+\phi_{+}^{2}(x) R_{0}\left(a_{+} / a_{-}\right)$is bounded by $C\left[1+\left(\phi_{+} \phi_{-}\right)^{2}\right]$.

Thus, to prove (3.23), we need to know that

$$
\int_{0}^{-\lambda / f} B(x) a x=O\left(|f|^{a}\right)
$$

and

$$
\int_{0}^{-\lambda / f}|B(x)|\left(\phi_{+} \phi_{-}\right)^{2} d x=O\left(|f|^{a}\right)
$$

In the region $\left[0, f^{-a}\right]$, where $B(x)=f x$ and $\phi_{+} \phi_{-}$is bounded, there is no problem since $\alpha<1 / 2$, so $\int f^{-\alpha} f x d x=O\left(f^{1-2 \alpha}\right)$. In the region $\left[f^{-\alpha},(-\lambda) / f\right]$, we have that

$$
\begin{aligned}
\left|\left(\phi_{+} \phi_{-}\right)(x)\right| & \leqslant C f^{-1 / 3} a(x, \lambda, f) b(x, \lambda, f) \\
& \leqslant \tilde{C} f^{-1 / 3}\left[1+f^{1 / 3}|x+\lambda / f|\right]^{-1 / 2}
\end{aligned}
$$

by (3.13)-(3.15) and the asymptotics of $a, b$. Therefore we break the interval $\left[f^{-\alpha},-\lambda f^{-1}\right]$ into two regions $R_{1}=\left[f^{-\alpha},-\frac{1}{2} \lambda f^{-1}\right]$ and $R_{2}=\left[-\frac{1}{2} \lambda f^{-1},-\lambda f^{-1}\right]$. In the region $R_{1}, \phi_{+} \phi_{-}$is bounded uniformly in $f$, so $\int_{R_{1}}\left|B(x) \| \phi_{+} \phi_{-}\right|^{2} d x$ $=O\left(f^{\alpha \epsilon}\right)$. In the region $R_{2},|B(x)|=O\left(|f|^{1+\epsilon}\right)$, so

$$
\begin{aligned}
\int_{R_{2}}|B(x)|\left|\phi_{+} \phi_{-}\right|^{2} d x & =O\left(f^{1+\epsilon}\right) \int_{0}^{-1 / 2 \lambda f} f^{-2 / 3}\left(1+f^{1 / 3} y\right)^{-1} d y \\
& =O\left(f^{\epsilon}\right) \int_{0}^{f^{-2 / 3}}(1-z)^{-1} d x \\
& =O\left(f^{\epsilon} \ln f\right) .
\end{aligned}
$$

Thus $X=O\left(|f|^{a}\right)$, proving (3.23).

Next, notice that from (3.21c), (3.20a), (3.20b), (3.15), and (3.17), we have

$$
\begin{aligned}
\operatorname{Im}\left(\frac{a_{+}}{a_{-}}\right)(x=-\lambda / f) & =-\frac{T}{2} \cdot\left(1+O\left(|f|^{a}\right)\right) \\
& =-\frac{1}{16}(-\lambda)^{-1 / 2} \exp \left(-\frac{4}{3}(-\lambda)^{3 / 2} / f\right) \cdot\left(1+O\left(|f|^{a}\right)\right) .
\end{aligned}
$$

Thus

$$
\operatorname{Im} G(f, \lambda)=-\frac{1}{16}(-\lambda)^{-1 / 2} \sigma_{+}(0, x) \exp \left(-\frac{4}{3}(-\lambda)^{3 / 2} / f\right) \cdot\left(1+O\left(|f|^{a}\right)\right) .
$$

Recalling that $\phi_{+}=\sigma_{+}$and $W\left\{\sigma_{-}, \sigma_{+}\right\}=2 \sqrt{-\lambda}, W\left\{\phi_{-}, \phi_{+}\right\}=1$, we see that

$$
G(f=0, \lambda)=\sigma_{-}(0, \lambda) / 2 \sqrt{-\lambda},
$$


and thus (3.24) and Theorem 2.5 imply (3.10) with

$$
\gamma=+\lambda_{1} 2\left(-\lambda_{0}\right)^{1 / 2}
$$

This agrees with the claimed formula if we note that first-order perturbation theory is known [26] to by asymptotic, so

$$
\lambda_{1}=-\left\langle\sigma_{-}, x \sigma_{-}\right\rangle /\left\langle\sigma_{-}, \sigma_{-}\right\rangle
$$

Proof of Proposition 3.2. We construct $b_{+}, b_{-}$by integrating the differential equation (2.6) from infinity and iterating the integral equation. The net result is

$$
\begin{gathered}
\left(\begin{array}{l}
b_{+} \\
b_{-}
\end{array}\right)=\sum_{j=0}^{\infty} M^{j}\left(\begin{array}{l}
0 \\
1
\end{array}\right) \\
M\left(\begin{array}{l}
g_{+} \\
g_{-}
\end{array}\right)(x)=\int_{\substack{x \\
\text { along } P_{2}}}^{\infty} \frac{V\left(x_{1}\right)}{W\left\{\eta_{-}, \eta_{+}\right\}}\left[\begin{array}{cc}
-\eta_{+} \eta_{-} & -\eta_{-}^{2} \\
\eta_{+}^{2} & \eta_{+} \eta_{-}
\end{array}\right]\left(\begin{array}{l}
g_{+}\left(x_{1}\right) \\
g_{-}\left(x_{1}\right)
\end{array}\right) d x_{1}
\end{gathered}
$$

where $M$ is a map on pairs of functions of $P_{2}$. Now introduce the norm on pairs of functions:

$$
\left\|\left(g_{+}, g_{-}\right)\right\| \equiv\left\|g_{-}\right\|_{\infty}+\left\|\eta_{-}^{-2} g_{+}\right\|_{\infty} .
$$

If we show that in the related operator norm $\|M\|=O\left(|f|^{a}\right)$, then we have convergence of the sum sufficiently strongly to verify that the limit solves the equation, and hence a proof of $(3.21 \mathrm{a}, \mathrm{b})$. We first note that for all $f$ and $\lambda$,

$$
\begin{aligned}
& \sup \left|\eta_{-}(z)^{-1} \eta_{-}(y)\right| \leqslant 1 \\
& |z|<|y| \\
& z, y \in P_{2}
\end{aligned}
$$

for by scaling the supremum is independent of $f$ and $\lambda$. The bound follows if we note that $C(z)$ is monotonic $(d A i(z) / d x<0$; see Appendix b). The point of (3.25) is that in estimating $\|M g\|$, we can replace $\eta_{-}(x)^{-2}=\left[\eta_{-}(x)^{-2} \eta_{-}\left(x_{1}\right)^{+2}\right]$ $\eta_{-}\left(x_{1}\right)^{-2}$ by $\eta_{-}\left(x_{1}\right)^{-2}$ inside the integral. Thus, by using the boundedness of $W\left\{\eta_{-}, \eta_{+}\right\}$for $f$ small, we need only show that each matrix element of

$$
\int_{\substack{-\lambda f^{-1} \\ \text { along } P_{2}}}|V(x)||N(x)| d x=O\left(|f|^{a}\right)
$$

where $N(x)$ is the matrix

$$
\left(\begin{array}{ll}
\eta_{-}^{-2} & 0 \\
0 & 1
\end{array}\right)\left(\begin{array}{ll}
-\eta_{+} \eta_{-} & -\eta_{-}^{2} \\
\eta_{+}^{2} & \eta_{+} \eta_{-}
\end{array}\right)\left(\begin{array}{ll}
\eta_{-}^{2} & 0 \\
0 & 1
\end{array}\right)=\left(\begin{array}{ll}
-\eta_{+} \eta_{-} & -1 \\
\left(\eta_{+} \eta_{-}\right)^{2} & \eta_{+} \eta_{-}
\end{array}\right) .
$$


Thus we only need that, for $p=0,1,2$ :

$$
\int_{0}^{\infty}\left(\left|\lambda f^{-1}\right|+|y|\right)^{-1-\epsilon}\left|f^{-1 / 3} g\left(f^{1 / 3} y\right)\right|^{p} d y=O\left(|f|^{a}\right)
$$

where

$$
g\left(f^{1 / 3} y\right)=\operatorname{Ai}\left(e^{-2 \pi i / 3} f^{1 / 3} y\right) A i\left(f^{1 / 3} y\right) .
$$

By the asymptotics in Appendix B, $|g(z)| \sim(4 \pi)^{-1}\left(z^{-1 / 2}\right)$, so

$$
|g(z)| \leqslant(1+z)^{-1 / 2}
$$

and $\left|f^{-1 / 3} g\left(f^{1 / 3} y\right)\right| \leqslant\left(f^{+2 / 3}+f y\right)^{-1 / 2}$. Changing $y$ to $f^{-1} z$ in (3.27), we obtain

$$
\text { (L.H.S. of }(3.27)) \leqslant f^{\epsilon} \int_{0}^{\infty}(|\lambda|+|z|)^{-1-\epsilon}\left(f^{2 / 3}+z\right)^{-p / 2} d z \text {. }
$$

For $p=0,1$, we bound $\left(f^{2 / 3}+z\right)^{-p / 2}$ by $z^{-p / 2}$ and obtain (3.27) with $a=\epsilon$. For $p=2$, we bound $\left(f^{2 / 3}+z\right)^{-1}$ by $z^{-1+\epsilon} f^{-2 \epsilon / 3}$, and obtain (3.27) with $a=\epsilon / 3$. We have thus proven (3.26), and therefore we have established the convergence of the expansion for $f$ small uniformly on $P_{2}$, as well as the estimates $(3.21 \mathrm{a}, \mathrm{b})$.

To prove (3.21c), we use the fact that we have just shown that $B_{-}^{(i)} \equiv \| i$ th term of $b_{-} \|_{\infty}$ is bounded by $C^{i}$ for a constant $C=O\left(|f|^{a}\right)$. Now let $B_{+}^{(i)} \equiv \| i$ th term of $b_{+} \|_{\infty}$ (note that there is no $\eta_{-}^{-1 / 2}$ as there is above). Then by the basic integral equation,

$$
B_{+}^{(i+1)} \leqslant X_{1} c^{i}+X_{2} B_{+}^{(i)}
$$

where

$$
\begin{aligned}
& X_{1}=\int_{\substack{-\lambda f^{-1} \\
\text { along } P_{2}}}\left|V(x) \eta_{-}^{2}(x)\right| d x, \\
& X_{2}=\int_{\substack{-\lambda f^{-1} \\
\text { along } P_{1}}}\left|V(x) \eta_{+}(x) \eta_{-}(x)\right| d x .
\end{aligned}
$$

We have already shown that $X_{2}=O\left(|f|^{a}\right)$. As for $X_{1}$, by changing variables as above,

$$
\begin{aligned}
X_{1} & \leqslant(\text { constant }) N^{2} \int_{0}^{\infty}\left(\left|\lambda f^{-1}\right|+|y|\right)^{-1-\epsilon}\left|A i\left(f^{1 / 3} y\right)\right|^{2} d y \\
& \leqslant\left(\text { constant } N^{2} f^{(2 / 3)+\epsilon}\right)=(\text { constant }) T f^{(1 / 3)+\epsilon}
\end{aligned}
$$

since $\int_{0}^{\infty}\left(A i(z)^{2} d z<\infty\right.$. Thus

$$
B_{+}^{(i+1)} \leqslant C_{1} T f^{1 / 3+\epsilon} c^{i}+D f^{a} B_{+}^{(i)},
$$


so for $f$ small (so that $D f^{a}<1$ and $c<1$ ),

$$
B_{+}=\sum_{i=1}^{\infty} B_{+}^{(w)} \leqslant\left(1-D f^{a}\right)^{-1} C_{1}(1-c)^{-1} T f^{1 / 3+\epsilon}
$$

i.e., (3.21c) holds with $a=1 / 3+\epsilon$.

Proof of Proposition 3.3. Since the proof is similar to the proof of the last proposition, we only sketch the details, emphasizing the differences. We pick an interval $[0, R]$ so that all the zeroes of $\sigma_{-}$lie in $[0, R]$ for $\lambda$ near $\lambda_{0}$. Thus $\phi_{-}$is nonvanishing on $[R,-\lambda / f]$ for all $f$ small, and we can use a norm $\|a\|=\left\|a_{-}\right\|_{\infty}+\left\|a_{+} \phi_{-}^{-2}\right\|_{\infty}$ on this interval. Moreover, $\sup \left[\phi_{-}(\gamma)\right]^{-1} \phi_{-}(x)$ is bounded uniformly in $\gamma, f$, and $x$. As in the proof of the foregoing proposition, one easily proves that in this norm the operator $M$ that solves the relevant integral equation is $O\left(|f|^{a}\right)$. Indeed, the basic estimate

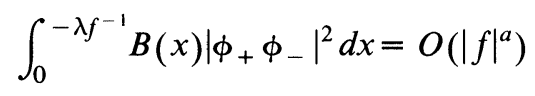

was already made in the proof of Theorem 3.1, above.

Of course, the data at $-\lambda / f$ must also be norm-bounded as $f \rightarrow 0$. Since $\phi_{-}^{-2}(-\lambda / f)$ diverges as $f \rightarrow 0$, there is something to check. The $a_{ \pm}(x=-\lambda / f)$ can be obtained from the $b_{ \pm}$constructed in the last proof and (3.19). By (3.20) and (3.21c), $a_{+}(x=-\lambda / f)=O\left(f^{1 / 3}\right) N^{2}$. Since $\phi_{-}(x=-\lambda / f)=B i(0) N^{2}$, we see that $\|a(-\lambda / f)\|$ is bounded as $f \rightarrow 0$. Thus the solution exists, and $a_{-}=2+O\left(|f|^{a}\right), a_{+}=O\left(|f|^{a}\right) \phi_{-}^{2}$ on $[R, \infty]$. This easily extends to the interval $(0, R)$ by integrating the equation without $\phi_{-}$"factored out."

Remark. In the proofs above, we had expansions which converged at an explicitly computable rate. Thus with finitely many computations we could compute to any given order $O\left(|f|^{m}\right)$.

Moreover, all the basic functions depending on $f$, i.e., $a, b$, and $c$ have known asymptotic expansions. Thus, in principle we could obtain $\operatorname{Im} \lambda(f)$ for any fixed $m$ with a (multiplicative) error of the form $\left(1+O\left(|f|^{m}\right)\right)$.

If the eigenfunction is cut off at large, real $x$, then the state that remains, while not an eigenfunction of the Hamiltonian, is a localized state of abnormally long decay time. Let $C(x)$ be a smoothed characteristic function of $[-\infty, 0]$, such that $C(x)=0$ if $x>1,=0$ if $x<-1$, and $C^{\prime}$ and $C^{\prime \prime}$ are bounded. Let $J$ be the exact eigenfunction of $H_{f}$ of (3.1)-(3.3) (or $h$ of (1.7) as in the following section) as a differential operator for $x \in \mathrm{C}$ with resonance boundary conditions, i.e., $H_{f} J=(E-i \epsilon) J$ where $\epsilon=O\left(\exp \left(-4\left(\lambda_{0}\right)^{3 / 2} / 3 f\right) \quad\left(\right.\right.$ resp. $O\left(f^{-1 /\left(-\lambda_{0}\right)^{1 / 2}}\right.$. $\exp \left(-4\left(-\lambda_{0}\right)^{3 / 2} / 3 f\right)$ ) has been explicitly calculated. (The sign of $\epsilon$ can be fixed by convention to be positive.) We have also seen that $J(x)$ and $J^{\prime}(x)$ are both uniformly $O\left(T^{1 / 2}\left\|\chi_{[0, t]}(x) J(x)\right\|\right)=O\left(\epsilon^{1 / 2}\left\|\chi_{[0, t]}(x) J(x)\right\|\right)$, for $x \in[t-1, t+1]$, where $t=-E / f$ is approximately the largest classical turning point and the $L^{2}$ 
norm is computed for real $x$. (Note that $\left\|\chi_{[0, t]} J\right\|$ is approximately the norm of the associated eigenfunction of the dilation-analytically continued version of $H_{f}$.) We then claim that $\Phi(x) \equiv C(x-t) J(x), x \in \mathrm{R}^{+}$, is a state of long sojourn in the sense of [33]: $\Phi$ is clearly an approximate eigenfunction of $H_{f}$, because the commutator $\left[H_{f}, C\right]=-C^{\prime \prime}-2 C^{\prime} d / d x$ is effectively bounded when acting on $J$

$$
\begin{aligned}
H \Phi & =C(x-t) H J-C^{\prime \prime} J-2 C^{\prime \prime} J \\
& =E \Phi+\delta \\
\delta & \equiv-i \epsilon \Phi-C^{\prime \prime}\left(\chi_{[t-1, t+1]} J\right)-2 C^{\prime}\left(\chi_{[t-1, t+1]^{\prime}}\right)
\end{aligned}
$$

is a vector whose norm is $O\left(T^{1 / 2}\left\|\chi_{[0, t]} J\right\|\right)$. That $\Phi$ has very slow decay is shown by differentiating the probability that the system stays in the same state:

$$
\begin{aligned}
d P_{t} / d t & \equiv \frac{d}{d t}|(\Phi, \exp (-i t H) \Phi)|^{2} \\
= & i\{(\Phi, \exp (-i t H) \Phi)(\Phi, \exp (i t H) H \Phi)-(\Phi, \exp (i t H) \Phi)(\Phi, \exp (-i t H) H \Phi)\} \\
= & i\{(\Phi, \exp (-i t H) \Phi)(\Phi, \exp (i t H)(E \Phi+\delta))-(\Phi, \exp (i t H) \Phi)(\Phi, \exp (-i t H)(E \Phi+\delta))\} \\
= & 2 \operatorname{Im}\{(\Phi, \exp (i t H) \Phi)(\exp (i t H) \Phi, \delta)\} \\
= & O(\|\delta\|) \text { by the Schwarz inequality for all times; and for short or } \\
& \times \text { intermediate times, it is } O((\Phi, \delta))=O(\epsilon)=O\left(\|\delta\|^{2}\right)=O(T) \cdot\left\|x_{[0, t]} J\right\| .
\end{aligned}
$$

§4. The Titchmarsh problem. In this section we discuss the asymptotics of the resonance problem associated with (1.7). We will concentrate on computing and controlling the asymptotics of the imaginary part of a Wronskian, since that is what we need in the following two sections. From this and calculations of the "unperturbed" Wronskians one can easily compute the imaginary part of the resonance eigenvalue for the Titchmarsh problem according to the analogue of Theorem 3.1.

There are two difficulties afflicting the present case, which were absent in the situation of the last section. First, the potential $V$ is not integrable. To get around this, we will have to use WKB comparison functions rather than the Airy functions of the last section in the region where $f x$ dominates $V$-except that near the classical turning point we will have to shift back to Airy functions (as usual with WKB calculations) to avoid the singularities of the WKB solutions at turning points. Thus we will have five regions instead of three.

Secondly, if $g$ is large, the comparison functions will be misbehaved at $x=0$. It was with this problem in mind that we divided the Wronskian by $a_{-}$in the last section. 
We will use similar symbols $\phi_{+}, \phi_{-}, A, B$, etc. to those of the last section with similar meanings. However, we reserve the right to change normalization conventions from the last section-we will, of course, be explicit about the normalizations used here.

We will deal with two solutions $R(x, f, \lambda)$ and $J(x, f, \lambda)$ of

$$
\left(-\frac{d^{2}}{d x^{2}}+g x^{-2}-x^{-1}-f x-\lambda\right) u(x)=0 .
$$

(We take $z=1$ and can later recover the $z$ dependence by scaling.) The regular solution $R$ will obey the boundary conditions at $x=0$, and $J$ will obey resonance conditions at infinity. As before, we will expand $J$ by variation of parameters in terms of two real-valued functions $\phi_{ \pm}$near $x=0$. The basic object that interests us is

$$
\begin{aligned}
G(f, \lambda) & =W\{J, R\} / a_{-}(x=1) \\
& =W\left\{\phi_{+}, R\right\}(x=1) \cdot\left(a_{+} / a_{-}\right)(x=1)+W\left\{\phi_{-}, R\right\}(x=1),
\end{aligned}
$$

and, in particular, since $\phi_{ \pm}$and $R$ are real-valued on the real axis,

$$
\operatorname{Im} G(f, \lambda)=W\left\{\phi_{+}, R\right\}(x=1) \cdot \operatorname{Im}\left(\frac{a_{+}}{a_{-}}\right)(x=1) .
$$

It will be useful to expand $R$ in terms of a function $\rho$ obeying

$$
\left(-\frac{d^{2}}{d x^{2}}+g x^{-2}-x^{-1}-\lambda\right) \rho(x)=0
$$

with Dirichlet boundary conditions; we also suppose that

$$
\lim _{x \rightarrow 0} \rho(x) / x^{1 / 2+\sqrt{g+1 / 4}}=1 .
$$

For special case $g=-3 / 16$, we will need the Neumann-like solution, which obeys

$$
\lim _{x \rightarrow 0}\left(\rho^{\prime}-(4 x)^{-1} \rho\right)=0, \quad \lim _{x \rightarrow 0} \rho x^{-1 / 4}=1 .
$$

Standard ordinary differential equations techniques show that since $[0,1]$ is a finite interval where $-f x$ is uniformly small,

$$
W\left\{\phi_{+}, R\right\}(x=1)=W\left\{\phi_{+}, \rho\right\}(x=1) \cdot(1+O(f)) .
$$

Since $\phi_{ \pm}$will also obey (4.3) near $x=1$, the Wronskians will be easy to compute. We thus want to write $\phi_{ \pm}$near zero in terms of the solutions of (4.3). 
These solutions are usually written as linear combinations of the Whittaker functions

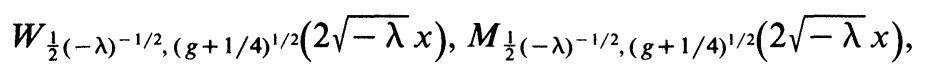

and

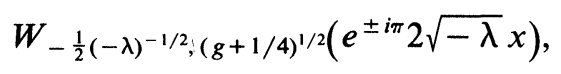

any two of which are linearly independent except at exceptional, discrete values of $\lambda$ and $g$. The analogues of the $\sigma_{ \pm}$of Section 3 are:

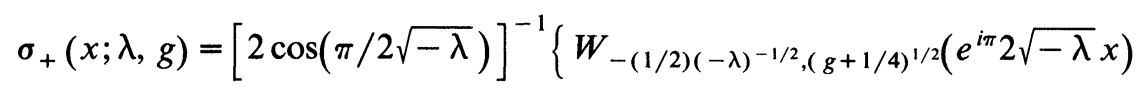

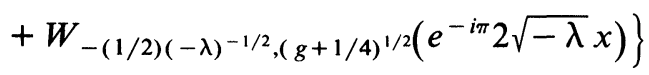

and

$$
\sigma_{-}(x, \lambda, g)=W_{(1 / 2)(-\lambda)^{-1 / 2},(g+1 / 4)^{1 / 2}(2 \sqrt{-\lambda} x)}
$$

For $\lambda$ near $-1 / 4 n^{2}$, which occurs at eigenvalues of (1.7) if $g=n^{2}-1 / 4,(4.6)$ is of the form $0 / 0$, and we make the different choice of (b.19b); on the real axis,

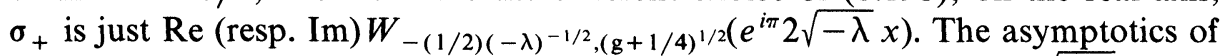
$\sigma_{ \pm}$, which because of the long-range $1 / x$ term are not simply $\exp ( \pm \sqrt{-\lambda} x)$, are described in Appendix B.

As mentioned in Section 2, we break the region $P_{1}=[1,-\lambda / f]$ into three regions, $\quad \mathrm{I}=\left[1, f^{-\alpha}\right], \quad \mathrm{II}=\left[f^{-\alpha},-\lambda \mathrm{f}^{-1}-f^{-\beta}\right], \quad$ and $\quad \mathrm{III}=\left[-\lambda f^{-1}-f^{-\beta}\right.$, $\left.-\lambda f^{-1}\right]$, where $\alpha \in(1 / 3,1 / 2)$ and $\beta \in(5 / 16,1)$. These mysterious choices are made so that later some integrals will converge. Also, as in Section 2, we write

$$
\begin{aligned}
& C(x) \equiv g x^{-2}-x^{-1}-f x-\lambda=A(x)+B(x), \text { where: } \\
& B(x)= \begin{cases}-f x & \text { in I } \\
C^{\prime \prime}[4 C]^{-1}-\frac{5}{16}\left[C^{\prime} / C\right]^{2} & \text { in II } \\
g x^{-2}-x^{-1} & \text { in III, }\end{cases}
\end{aligned}
$$

and pick $\phi_{ \pm}$to be $C^{1}$ on $P_{1}$, obeying

$$
-\phi_{ \pm}^{\prime \prime}+A(x) \phi_{ \pm}=0
$$

In the region I, $\phi_{ \pm}$are linear combinations of $\sigma_{ \pm}$, and in region III, combinations of the Airy functions $a(x ; f, \lambda)$ and $b(x ; f, \lambda)$ used in Section 3 . The rather complicated form for $B$ in Region II is such that the solutions of (4.8) 
are linear combinations of the two WKB solutions:

$$
\chi_{ \pm}(x ; f, \lambda) \equiv(C(x, F))^{-1 / 4} \exp \left( \pm \int_{t_{1}}^{x}(C(y ; f))^{1 / 2} d y\right),
$$

where $t_{1}$ is the smallest root of $C(x ; f)=0$ with $C^{\prime}>0$ (as $f \rightarrow 0, t_{1} \rightarrow$ $\left.(-2 \lambda)^{-1}(1+\sqrt{1+4 \lambda g})\right)$.

We choose $\phi_{ \pm}$so that

$$
\begin{aligned}
& \phi_{+}=\sigma_{+} \text {in Region I } \\
& \sigma_{-}=N b \text { in Region III, }
\end{aligned}
$$

where $N$ is a constant (in $x$ ) such that $W\left\{\phi_{-}, \phi_{+}\right\}=1$. The rather tedious construction of $\phi_{ \pm}$in the other regions by matching at the boundaries is to be found in Appendix C. In terms of the tunneling factor (computed in Appendix A)

$$
T(f, \lambda) \equiv \exp \left(-2 \int_{t_{1}}^{t_{2}}(C(x))^{1 / 2} d x\right),
$$

where $t_{2}$ is the root of $C=0$ near $x=-\lambda / f$, we find that:

$$
N=\frac{\sqrt{\pi}}{2 \gamma_{+}} f^{-1 / 6} T^{1 / 2} \cdot\left(1+O\left(f^{\epsilon}\right)\right),
$$

where

$$
\gamma_{+}=(-\lambda)^{1 / 4}\left[\frac{2 e \sqrt{-\lambda}}{\sqrt{1+4 g \lambda}}\right]^{1 / 2 \sqrt{-\lambda}}\left[\frac{(2 \sqrt{-\lambda}-1)(1+\sqrt{1+4 g \lambda})}{-4 \lambda g-1-\sqrt{1+4 g \lambda}}\right]^{\sqrt{g}} .
$$

Moreover, in Region I:

$$
\begin{aligned}
& \phi_{+}=\sigma_{+} \\
& \phi_{-}=(2 \sqrt{-\lambda})^{-1} \sigma_{-} \cdot\left(1+O\left(f^{\epsilon}\right)\right)
\end{aligned}
$$

and in Region II:

$$
\begin{aligned}
& \phi_{+}=\gamma_{+} \chi_{+} \cdot\left(1+O\left(f^{\epsilon}\right)\right) \\
& \phi_{-}=\frac{1}{2 \gamma_{+}} \chi_{-} \cdot\left(1+O\left(f^{\epsilon}\right)\right)
\end{aligned}
$$

and in Region III:

$$
\begin{aligned}
& \phi_{+}=\left[\pi f^{-1 / 3} / N\right] a(x ; f, x) \cdot\left(1+O\left(f^{\epsilon}\right)\right) \\
& \phi_{-}=N b(x ; f, \lambda) .
\end{aligned}
$$


We will break the second part of the path going to infinity, $\left\{x=-\lambda f^{-1}+\right.$ $\left.r e^{i \pi / 3} \mid r>0\right\}$, into two pieces, Region IV, where $\left|x+\lambda f^{-1}\right| \leqslant f^{-\beta}$, and Region $\mathrm{V}$, where $\left|x+\lambda f^{-1}\right| \geqslant f^{-\beta}$. In Region IV, $B$ is the same as in Region III, so that the comparison functions $\eta_{ \pm}$are sums of the Airy functions $a(x ; f, \lambda)$ and $c(x ; f, \lambda)$. In Region $\mathbf{V}, B$ is the same as in Region II, so that $\eta_{ \pm}$are sums of WKB solutions $\chi_{ \pm}$. The functions $\chi_{ \pm}$are extended to complex $x$ by placing branch cuts on $(-\lambda / f, \infty)$ and from $t_{1}$ along the real axis to $-\infty$. Thus

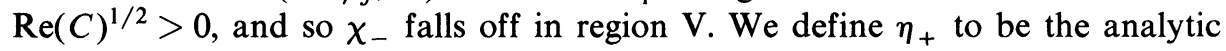
continuation of $\phi_{+}$into Region IV, and then extend it by continuity to Region $\mathrm{V}$, and we define

$$
\eta_{-} \equiv\left[\pi^{-1 / 2} f^{1 / 6} T^{-1 / 2} N\right] \chi_{-}
$$

in Region V. Calculations of Appendix C show that in Region IV:

$$
\begin{aligned}
& \eta_{+}=\left(2 \pi^{1 / 2} f^{-1 / 6} T^{-1 / 2} \gamma_{+}\right) a(x ; f, \lambda) \cdot\left(1+O\left(f^{\epsilon}\right)\right), \\
& \eta_{-}=\pi^{1 / 2} f^{-1 / 6} \gamma_{+}^{-1} T^{1 / 2} e^{+\pi i / 6} c(x ; f, \lambda) \cdot\left(1+O\left(f^{\epsilon}\right)\right) ;
\end{aligned}
$$

and in Region V:

$$
\begin{aligned}
& \eta_{+}=\gamma_{+} \chi_{+} \cdot\left(1+O\left(f^{\epsilon}\right)\right) \\
& \eta_{-}=\left(2 \gamma_{+}\right)^{-1} \chi_{-} \cdot\left(1+O\left(f^{\epsilon}\right)\right) .
\end{aligned}
$$

We now turn to the proof of the convergence of the variation-of-parameters construction of the resonance solution $J$.

As in the last section, we work with a weighted norm. However, the norm $\left\|\left(g_{+}, g_{-}\right)\right\|=\left\|g_{-}\right\|_{\infty}+\left\|\eta_{-}^{-2} g_{+}\right\|_{\infty}$ used there will not work (see below), and instead we use the norm

$$
\left\|\left(g_{+}, g_{-}\right)\right\|=\left\|g_{-}\right\|_{\infty}+f^{-\Delta}\left\|\eta_{-}^{-2} g_{+}\right\|_{\infty},
$$

where $\Delta$ is chosen to obey:

$$
0<\Delta<\min (\beta, 1-\beta)=1-\beta .
$$

The net effect is to change the matrix $N$ so that it becomes

$$
\left[\begin{array}{ll}
-\eta_{+} \eta_{-} & -f^{-\Delta} \\
f^{\Delta}\left(\eta_{+} \eta_{-}\right)^{2} & \eta_{+} \eta_{-}
\end{array}\right)
$$

Tr us we need to know two facts to get the convergence of the variation of parameters along $P_{2}$ :

$$
\sup _{\substack{z, y \in P_{2} \\|z|<|y|}}\left|\eta_{-}(z)^{-1} \eta_{-}(y)\right|<\infty
$$


uniformly in $f$ small and $\lambda$ near $\lambda_{0}$; and

$$
\int_{P_{2}} B(x) f^{\Delta(p-1)}\left(\eta_{+} \eta_{-}\right)^{p} d x=O\left(|f|^{a}\right)
$$

for $p=0,1,2$. (4.16) is easy since $\eta_{-}$is monotonic in Region $V\left(\chi_{-}\right.$is monotonic), and up to multiplicative errors of the form $\left(1+O\left(f^{\epsilon}\right)\right)$ it is monotonic in Regions IV ( $c$ is monotonic). To check (4.17) we consider the contributions of Regions IV and V separately.

In Region $\mathrm{V}$, in terms of the variable $r=e^{i \pi / 3}(x+\lambda / f), C(x, f)=g x^{-2}-$ $x^{-1}-f r e^{i \pi / 3}$ obeys $|C| \geqslant f\left(r-r_{0}\right)$, since $\left|x^{-1}\right| \leqslant c f$ on all of $P_{2}$. Since $\left|C^{\prime}\right| \leqslant$ const. $f,\left|C^{\prime \prime}\right| \leqslant O\left(x^{-3}\right)=O\left(f^{2} r^{-1}\right)$ and $r-r_{0} \geqslant r / 2$ in Region $\mathrm{V}$, we see that

$$
|B(x)| \leqslant \text { const } \cdot r^{-2} \quad(\text { Region } \mathrm{V}) .
$$

Now $\eta_{+} \eta_{-}=O\left(C^{-1 / 2}\right) \leqslant O\left((f r)^{-1 / 2}\right)$, so in Region $\mathrm{V}$, (4.17) follows from $f^{\Delta(p-1)} \int_{f^{-\beta} r^{-2}}^{\infty}(f r)^{-p / 2} d r=O\left(f^{a}\right)$ for $p=0,1,2$. This is easy to see because $\beta>5 / 6(\beta>1 / 2$ would do), and $\Delta<\beta$.

For Region IV, we mimic the estimates following (3.27) and see that we will need to control

$$
A_{p} \equiv f^{\Delta(p-1)} \int_{0}^{f^{1-\beta}}(|\lambda|+r)^{-1}\left(f^{2 / 3}+r\right)^{-p / 2} d r
$$

For all values of $p$ we can estimate $(|\lambda|+r)^{-1}$ above by $|\lambda|^{-1}$. For $p=0,1$, we estimate $\left(f^{2 / 3}+r\right)^{-p / 2}$ by $r^{-p / 2}$ and see that $A_{0}=O\left(f^{1-\beta-\Delta}\right)$, which is $O\left(f^{a}\right)$ since $\Delta<1-\beta$, and that $A_{1}=O\left(f^{(1 / 2)(1-\beta)}\right)$. To treat $A_{2}$, notice that

$$
\begin{aligned}
\int_{0}^{f^{1-\beta}}\left(f^{2 / 3}+r\right)^{-1} d r & =\ln \left[\left(f^{2 / 3}+f^{1-\beta}\right) / f^{2 / 3}\right] \\
& =O(\ln f),
\end{aligned}
$$

since $1-\beta<2 / 3$. Thus $A_{2}=O\left(f^{\Delta} \ln f\right)$, which is $O\left(f^{a}\right)$ since $\Delta>0$.

This completes the proof of (4.16). Notice that if we had not used the $f^{-\Delta}$ weighting in the norm, we would have had a $\ln f$ type divergence.

The estimates (4.15), (4.16) prove the first part of:

Proposition 4.1. There is a solution $J$ of (4.1) along the curve $P_{2}$ with

$$
b_{-}=1+O\left(|f|^{a}\right)
$$

for some $a>0$, uniformly in $x \in P_{2}$, and

$$
b_{+}=O\left(\eta_{-}^{2}\right) \cdot O\left(|f|^{a+\Delta}\right)
$$


Moreover,

$$
b_{+}(-\lambda / f)=\frac{T}{\gamma_{+}^{2}} \cdot O\left(|f|^{a}\right)
$$

Proof. We need only check (4.19b). As in the proof of Proposition 3.2, we only need to show that

$$
\begin{gathered}
\int\left|B(x) \eta_{+}(x) \eta_{-}(x)\right| d x=O\left(|f|^{a}\right), \\
\int\left|B(x) \eta_{-}^{2}(x)\right| d x=\frac{T}{\gamma_{+}^{2}} \cdot\left(O|f|^{a}\right) .
\end{gathered}
$$

(4.20) is just (4.16) for $p=1$. We check (4.21) in Regions IV and V separately. In Region IV:

$$
\begin{aligned}
\int_{\text {Region IV }}\left|B(x) \eta_{-}(x)^{2}\right| d x \\
=\left.O\left(T / \gamma_{+}^{2}\right) f^{-1 / 3} \int_{0}^{f^{-\beta}} A i\left(f^{1 / 3} y\right)\right|^{2}\left(\left|\lambda f^{-1}\right|+|y|\right)^{-1} d y \\
=O\left(T / \gamma_{+}^{2}\right) \cdot O\left(f^{2 / 3}\right) \int_{0}^{\infty}(A(i x))^{2} d x
\end{aligned}
$$

so the Region IV contribution to the integral obeys (4.21). As for the contribution from Region $\mathrm{V}$, since $\eta_{-}=\left(2 \gamma_{+}\right)^{-1} \chi_{-}\left(1+O\left(|f|^{\epsilon}\right)\right)$, we need that

$$
\int_{(\text {Region V) }}\left|B(x) \chi_{-}^{2}(x)\right| d x=T \cdot O\left(|f|^{a}\right) \text {. }
$$

But $\chi_{-}^{2}$ involves $\exp \left(-2 \int_{t_{1}}^{x} \cdots\right)$, and since $\operatorname{Re}\left(\int_{t_{2}}^{x} \cdots\right) \geqslant 0$, a factor of $T$ comes out and thus (4.22a) follows from

$$
\int_{(\text {Region V) }}\left|(C(x))^{-1 / 2} B(x)\right| d x=O\left(|f|^{a}\right) .
$$

But by (4.18), $B(x)=O\left(r^{-2}\right)$ and $C^{-1 / 2}=O(f r)^{-1 / 2}$, so the left side of (4.22b) is $O\left(f^{(3 \beta-1) / 2}\right)$, which is $O\left(f^{\epsilon}\right)$ since $\beta>1 / 3$.

The formulae transforming from $b_{ \pm}$to $a_{ \pm}$are identical to those in the short-range case, i.e., (3.20a). Since $W\left\{\eta_{-}, \phi_{-}\right\} \sim\left(T / \gamma_{+}^{2}\right) \cdot($ const.), the leading term is from $W\left\{\eta_{-}, \phi_{-}\right\}$on account of (4.19b). Thus:

$$
\operatorname{Im}\left(\frac{a_{+}}{a_{-}}\right)(x=-\lambda / f)=-\operatorname{Im} \frac{W\left\{\eta_{-}, \phi_{-}\right\}}{W\left\{\eta_{-}, \eta_{+}\right\}} \cdot\left(1+O\left(|f|^{a}\right)\right)=\frac{T}{4 \gamma_{+}^{2}} \cdot\left(1+O\left(|f|^{a}\right)\right)
$$

by $(4.12 c),(4.13 a),(4.14 a)$, and (b.8). 
Next we use (3.19) and the estimate $(4.19 \mathrm{~b})$ on $b_{+}(-\lambda / f)$ to find that

$$
\begin{aligned}
a_{+}(-\lambda / f) & =O\left(T / \gamma_{+}^{2}\right) \\
& =\phi_{-}(-\lambda / f)^{2} \cdot O\left(f^{1 / 3}\right)
\end{aligned}
$$

by (4.9).

The point of (4.24) is that it asserts that if we use the norm

$$
\left\|\left(g_{+}, g_{-}\right)\right\|=\left\|g_{-}\right\|_{\infty}+f^{-\Delta}\left\|\phi_{-}^{2} g_{+}\right\|_{\infty},
$$

then the "initial data" $\left(a_{+}(-\lambda / f), a_{-}(-\lambda / f)\right)$ for integrating the integral equation have finite norm so long as $\Delta<1 / 3$. For this reason, the variation of parameters along $P_{1}$ will converge so long as for some $R_{0}>0$ independent of $f$,

$$
\sup _{\substack{x, y \in P_{1} \\ R_{0} \leqslant|z| \leqslant y}}\left|\phi_{-}(z)^{-1} \phi_{-}(y)\right|<\infty,
$$

and

$$
\int_{P_{1}} B(x) f^{\Delta(p-1)}\left|\phi_{+} \phi_{-}\right|^{p} d x=O\left(|f|^{a}\right)
$$

The condition $z>R_{0}$ can be imposed since on $\left[0, R_{0}\right]$ we can use an unweighted norm as we did in the short-range case. (4.25) is easy to show, since up to errors $1+O\left(f^{\epsilon}\right), \phi_{-}$is monotonic in $\left(R_{0},-\lambda / f\right)$, where $R_{0}$ is chosen so that $\sigma_{-}$is monotonic past $R_{0}$ (this can be done since $R_{0}$ can be chosen with $\sigma_{-}$convex on $\left.\left(R_{0}, \infty\right)\right)$. This leaves only (4.26). As usual we prove it separately in Regions I-III.

In Region II, we write

$$
C(x, f)=g x^{-2}-x^{-1}+f r ; \quad r=-(x+\lambda / f) .
$$

We first note that

$$
C \geqslant \frac{1}{2} f\left(r-r_{0}\right) \text { in Region II; }
$$

for if $r<-\frac{1}{2} \lambda / f$, then $\left|g x^{-2}-x^{-1}\right| \leqslant O(f) \leqslant f r_{0}$. If $r>-\frac{1}{2} f^{-1} \lambda$ but $x>f^{-\alpha}$, then $\left|g x^{-2}-x^{-1}\right| \leqslant O\left(f^{\alpha}\right)<(1 / 2) f r$. Next notice that in all of Region II, $C^{\prime}=f+x^{-2} \leqslant O\left(f^{2 \alpha}\right)$, so, since $\phi_{+} \phi_{-}=O\left(C^{-1 / 2}\right)$,

$$
\begin{aligned}
\int_{\mathrm{II}}\left(\frac{C^{\prime}}{C}\right)^{2}\left(\phi_{+} \phi_{-}\right)^{p} f^{\Delta(p-1)} \\
\quad \leqslant O\left(\int_{f^{-\beta}}^{\infty} f^{\Delta(p-1)}(f r)^{-2-p / 2} f^{4 \alpha}\right) \\
\quad=O\left(f^{\gamma(p)}\right),
\end{aligned}
$$


where:

$$
\begin{aligned}
& \gamma(0)=\beta+4 \alpha-2-\Delta \\
& \gamma(1)=(3 / 2) \beta+4 \alpha-5 / 2 \\
& \gamma(2)=\Delta+2 \beta+4 \alpha-3
\end{aligned}
$$

All these $\gamma$ 's are positive so long as $\Delta<1 / 6$ since $\alpha>1 / 3, \beta>5 / 6$. To control

$$
\int_{\text {II }} \frac{C^{\prime \prime}}{C}\left(\phi_{+} \phi_{-}\right)^{p} f^{\Delta(p-1)},
$$

we break the integral into two pieces, for $x$ from $f^{-\alpha}$ to $-\lambda / 2 f$, and for $r$ from $f^{-\beta}$ to $-\lambda / 2 f$. In the first piece $C \geqslant$ constant by (4.28) so we have to bound

$$
\int_{f^{-\alpha}}^{-\lambda / 2 f} f^{\Delta(p-1)} x^{-3} d x=O\left(f^{2 \alpha+(p-1) \Delta}\right)
$$

(since $C^{\prime \prime}=O\left(x^{-3}\right)$ ), and this is small if $\Delta<2 \alpha$. In the second piece, since $C^{\prime \prime} \leqslant f^{3}$, we have to bound

$$
f^{3} \int_{f^{-\beta}}^{f^{-1}} f^{\Delta(p-1)}(f r)^{-1-p / 2} d r
$$

which is $O\left(f^{2-\Delta} \ln f\right), O\left(f^{3 / 2} f^{\beta / 2}\right), O\left(f^{1+\Delta} f^{\beta}\right)$ for $p=0,1,2$. Since all these $\rightarrow 0$, we have verified (4.26) in Region II.

Finally, in Region I we note that on $[1, \infty), \phi_{+} \phi_{-}$is uniformly bounded by (b.18) and (b.22), so that (4.26) requires that

$$
\int_{0}^{f^{-\alpha}}(f r) f^{\Delta(p-1)} d r=(1 / 2) f^{1-2 \alpha} f^{\Delta(p-1)},
$$

which $\rightarrow 0$ if $\Delta<1-2 \alpha$. Thus, since $\alpha \in(1 / 3,1 / 2), \beta \in(5 / 6,1)$, (4.26) will hold if $0<\Delta<1 / 6, \Delta<1-2 \alpha$ and $\Delta<1-\beta$. We have thus proven the first part of

Proposition 4.2. The solution $J$ given by Proposition 4.1 can be continued along $P_{1}$ by variation of parameters, and

$$
\operatorname{Im}\left(\frac{a_{+}}{a_{-}}\right)(x=1)=\frac{T}{4 \gamma_{+}^{2}} \cdot\left(1+O\left(|f|^{\epsilon}\right)\right) .
$$

Proof. By Theorem 2.6, we must show that the quantity $X$ of $(2.11)$ is $O\left(|f|^{\epsilon}\right)$. But since $a_{-}=1+O\left(|f|^{\epsilon}\right)$ by our basic formulae and $a_{+}=O\left(\phi_{-}^{2}\right)$, we see that

$$
|X| \leqslant \text { const. } \int_{P_{1}}|B(x)|\left[1+\left(\phi_{+} \phi_{-}\right)^{2}\right] d x=O\left(|f|^{\epsilon}\right) \quad \text { by (4.26). }
$$


Combining (4.2), (4.5), (4.10) and the formula for $T$ computed in Appendix A with the last proposition we find:

TheOREM 4.3. Let $G(g, f, \lambda)$ be the Wronskian, normalized by $a_{-}(x=1)$, of the two solutions $J$ and $R$ of (4.1). Then for $g$ and $\lambda$ fixed,

$$
\operatorname{Im} G=(1 / 4) W\left\{\sigma_{+}, \rho\right\} \frac{T}{\gamma_{+}^{2}} \cdot\left(1+O\left(|f|^{\epsilon}\right)\right),
$$

where $\rho$ is given by (4.4) (or $(4.4 \mathrm{~N})$, depending on the boundary conditions at 0$)$, and

$$
T / \gamma_{+}^{2}=(-\lambda)^{-1 / 2}\left[\frac{8(-\lambda)^{3 / 2}}{f}\right]^{1 /(-\lambda)^{1 / 2}} \exp \left[-4(-\lambda)^{3 / 2} / 3 f\right]
$$

In the next two sections we will be interested in special cases $g=-3 / 16$ and $g=\left(m^{2} / 4-1 / 4\right), m=0,1,2, \ldots$ To avoid rather complicated-looking expressions, we will compute the Wronskians only in these cases, although it would be easy to carry the $g$ along in general. These formulae are equivalent to a computation of the asymptotics of the Titchmarsh resonance eigenvalues.

§5. The Bender-Wu formula. In this section our goal is to prove the following result:

THEOREM 5.1. Let $\Lambda_{k}(\beta)$ be the analytic continuation of the kth eigenvalue $(k=1,2, \ldots)$ of $-d^{2} / d v^{2}+v^{2}+\beta v^{4}$ to the cut plane $\mathrm{C} \backslash(-\infty, 0]$. Then as $\mu \downarrow 0$,

$$
\operatorname{Im} \Lambda_{k}(-\mu+i O)=2^{3 k-1}[(k-1) ! \sqrt{\pi}]^{-1} \mu^{-k+1 / 2} \exp (-2 / 3 \mu) \cdot\left(1+O\left(\mu^{\alpha}\right)\right)
$$

for some $\alpha>0$. In particular, (1.11) and thus also (1.9) hold.

We note that Bender and $\mathrm{Wu}[6]$ obtained (5.1) (without rigor) for general $k$. It turns out that we have to prove (5.1) separately for $k$ even and $k$ odd, corresponding to Dirichlet and Neumann conditions at $x=0$. We first consider the case of Dirichlet boundary conditions, i.e., $k=2 n$.

Proposition 5.2. Let $H(\tilde{f}, \tilde{\lambda}, z)$ be the normalized Wronskian of the resonance solution at infinity and the Dirichlet solution at zero for the Titchmarsh problem

$$
\left(-\frac{d^{2}}{d x^{2}}-\frac{3}{16} x^{-2}-z x^{-1}-\tilde{f} x-\tilde{\lambda}\right) u=0 .
$$

Fix $\tilde{\lambda}_{0}=-1 / 4$ and $z_{0}=n-1 / 4, n=1,2, \ldots$, so that $H\left(\tilde{f}=0, \tilde{\lambda}_{0}, z_{0}\right)=0$. Then for $\tilde{\lambda}_{0}$ fixed at $-1 / 4$ and $\tilde{f}$ near zero and positive, there is a unique solution 
$z(\tilde{f})$ of $H(\tilde{f}, \tilde{\lambda}, z)=0$ with

$$
\operatorname{Im} z \sim-2^{2 n-2}[(2 n-1) ! \sqrt{\pi}]^{-1}(\tilde{f})^{-2 n+1 / 2} \exp (-1 / 6 \tilde{f})
$$

Before proving Proposition 5.2, we note that it implies Theorem 5.1 for $k$ even:

Proof of Theorem 5.1 for $k$ even. Let $k=2 n$. By the basic transmutation formulae of Propositions 1.1 and 2.3, $\operatorname{Im} \Lambda_{k=2 n}(-\mu+i O)=-4 \operatorname{Im} z(\tilde{f}=\mu / 4)$ for the solution with $\tilde{\lambda}=-\frac{1}{4} \alpha=-\frac{1}{4} \quad(\alpha=1)$ and $z_{0}=+\frac{1}{4} \Lambda_{k=2 n}(\mu=0)$ $=\frac{1}{4}(2 k-1)=n-\frac{1}{4}$. (The minus sign in $\operatorname{Im} \Lambda_{k}=-4 \operatorname{Im} z$ comes from the facts that, by Proposition 2.3, $4 \operatorname{Im} z=\operatorname{Im} \Lambda(-\mu+i O)$ and that $\operatorname{Im} \Lambda(-\mu-i O)=$ $-\operatorname{Im} \Lambda(-\mu+i O)$.) Substituting $\mu / 4$ for $\tilde{f}$ and $\frac{1}{2} k$ for $n$ in (5.2) and multiplying by -4 yields $(5.1)$.

Proof of Proposition 5.2. We begin by observing that by scaling

$$
H(\tilde{f}, \tilde{\lambda}, z)=G\left(z^{-3} \tilde{f}, z^{-2} \tilde{\lambda}\right),
$$

Where $G(f, \lambda)$ is the Wronskian for $z=1$ discussed in $\S 4$. Thus, noting that

$$
\operatorname{Im} H(\tilde{f}, \tilde{\lambda}, z)=-\exp \left(-4(-\tilde{\lambda})^{3 / 2} / \tilde{f}\right)
$$

has an exponential factor independent of $z$, we see that, by Theorem 2.5,

$$
\begin{gathered}
\operatorname{Im} z(f) \sim-\operatorname{Im} H(\tilde{f}, \tilde{\lambda}, z(f)) / \frac{\partial H}{\partial z}\left(\tilde{f}=0, \tilde{\lambda}, z_{0}\right) \\
=\operatorname{Im} G\left(z^{-3} f, z^{-2} \tilde{\lambda}\right) / 2 z^{-3} \tilde{\lambda} \frac{\partial G}{\partial \lambda}\left(0, z^{-2} \lambda\right) \\
=\left(\frac{z_{0}}{2 \tilde{\lambda}}\right)^{3} \frac{W\left\{\sigma_{+}, \rho\right\}}{\frac{\partial}{\partial \lambda}\left[W\left\{\sigma_{-}, \rho\right\} / 2(-\sqrt{\lambda})\right]} \frac{T}{4 \gamma_{+}^{2}},
\end{gathered}
$$

all evaluated at $f=z^{-3} \tilde{f}$ and $\lambda=z^{2} \tilde{\lambda}$. To get (5.4) we need to note that since $G(f, \lambda)=O\left(f^{a}\right)$ by our method, implying that $\partial G / \partial f=O\left(f^{a-1}\right)$, we have that $3 \tilde{f} z^{-4}\left(\partial G\left(\tilde{f} z^{-3}\right)\right) / \partial f$ goes to zero with $\tilde{f}$. Equation (5.5) follows from Theorem 4.3. For later purposes, we remark that (5.5) also holds in the Neumann case, except that $\rho$ changes.

To evaluate the Wronskians in (5.5), we can write that $W\left\{\sigma_{ \pm}, \rho\right\}$ $=\lim _{x \rightarrow 0} \sigma_{ \pm}(x) / x^{1 / 4}$ by (4.4) and (4.5) $(g=-3 / 16$ and suitably normalized $\rho)$. That is, $\sigma_{ \pm}$is a multiple of the regular solution $\rho\left(\sim x^{3 / 4}\right)$ if and only if the dominant $x^{1 / 4}$ term is missing. Thus, by (b.17),

$$
W\left\{\sigma_{-}, \rho\right\}=\Gamma\left(\frac{1}{2}\right)(2 \sqrt{-\lambda})^{1 / 4} / \Gamma\left(\frac{3}{4}-\frac{1}{2 \sqrt{-\lambda}}\right)
$$


and by (b.21),

$$
W\left\{\sigma_{+}, \rho\right\}=\left\{\Gamma\left(\frac{1}{2}\right)(2 \sqrt{-\lambda})^{1 / 4} / \Gamma\left(\frac{3}{4}+\frac{1}{2 \sqrt{-\lambda}}\right)\right\} \frac{\cos (\pi / 4)}{\cos (\pi / 2 \sqrt{-\lambda})}
$$

To evaluate when $W\left\{\sigma_{-}, \rho\right\}=0$, note that this can only happen when the denominator of (5.6) has a pole, i.e., at

$$
\begin{aligned}
\frac{3}{4}-\frac{1}{2 \sqrt{-\lambda_{0}}} & =-(n-1), \quad n=1,2, \ldots, \text { or } \\
\lambda_{0}^{(n)} & =-\frac{1}{4(n-1 / 4)^{2}} .
\end{aligned}
$$

By the relation $\lambda_{0}=\tilde{\lambda}_{0} z^{-2}$ we see that for $\tilde{\lambda}_{0}=-\frac{1}{4}$, this corresponds to $z_{0}=n-1 / 4$. At this value of $\lambda_{0}$,

$$
W\left\{\sigma_{+}, \rho\right\}=\frac{\Gamma\left(\frac{1}{2}\right)\left(2 \sqrt{-\lambda_{0}}\right)^{1 / 4}}{\Gamma\left(n+\frac{1}{2}\right)} \cdot(-1)^{n}
$$

since $\cos (\pi(n-1 / 4))=\cos (-n \pi+\pi / 4)=(-1)^{n} \cos (\pi / 4)$. On the other hand, since $\left.[\partial(1 / \Gamma(x)) / \partial x]\right|_{x=-(n-1)}=(n-1) !(-1)^{n-1}$ at that value of $\lambda_{0}$,

$$
\frac{\partial W}{\partial \lambda}\left\{\sigma_{-}, \rho\right\}=(-1)^{n} 2 \Gamma\left(\frac{1}{2}\right)(2 \sqrt{-\lambda})^{1 / 4-3}(n-1) !
$$

Finally, by (4.30),

$$
\frac{T}{\gamma_{+}^{2}}\left(z_{0}^{-3} \tilde{f}, z_{0}^{-2} \tilde{\lambda}_{0}\right)=\left(-\tilde{\lambda}_{0}\right)^{-1 / 2} z_{0}\left[\frac{8\left(-\tilde{\lambda}_{0}\right)^{3 / 2}}{\tilde{f}}\right]^{2 n-1 / 2} \exp \left(-\frac{4(-\tilde{\lambda})^{3 / 2}}{3 \tilde{f}}\right)
$$

at $z^{-2} \lambda_{0}=\lambda=-1 / 4(n-1 / 4)^{2}$. Combining the last three formulae with (5.5) and using $(n-1) ! \Gamma(n+1 / 2)=2^{-(2 n-1)}(2 n-1) ! \sqrt{\pi}, \lambda_{0}=-1 / 4, z_{0}=n-1 / 4$, we find that (5.2) holds.

As in the case of $k$ even, the result for $k$ odd follows from:

Proposition 5.3. If $\tilde{H}(\tilde{f}, \tilde{\lambda}, z)$ is the normalized Wronskian for the same equation as in Proposition 5.2, but involving the Neumann and resonance solutions, then for $\tilde{\lambda}_{0}=-\frac{1}{4}$ and $z_{0}=n-\frac{3}{4}, n=1,2, \ldots\left(\right.$ so that $\left.\tilde{H}\left(\tilde{f}=0, \tilde{\lambda}_{0}, z_{0}\right)=0\right)$,

$$
\operatorname{Im} z \sim-2^{2 n-3}[(2 n-2) ! \sqrt{\pi}]^{-1}(\tilde{f})^{-2 n+3 / 2} \exp (-2 / 6 \tilde{f}) .
$$

Proof. As noted in the proof of Proposition 5.2, Equation (5.5) holds in this case also, but now $\rho$ obeys $(4.4 \mathrm{~N})$. Thus if $\sigma_{ \pm} \sim c_{ \pm} x^{1 / 4}+d_{ \pm} x^{3 / 4}+\cdots$, then 
we find that:

$$
W\left\{\sigma_{ \pm}, \rho\right\}=\lim _{x \rightarrow 0}\left((1 / 4) \sigma_{ \pm} x^{-3 / 4}-\sigma_{ \pm}^{\prime} x^{1 / 4}\right)=-1 / 2 d_{ \pm} .
$$

Thus, by (b.17') and (b.21') we get:

$$
\begin{aligned}
& W\left\{\sigma_{+}, \rho\right\}=\frac{2^{-1 / 4}(-\lambda)^{3 / 8} \sqrt{\pi} \cos (\pi / 4)}{\Gamma\left(\frac{1}{4}+\frac{1}{2 \sqrt{-\lambda}}\right) \cos (\pi / 2 \sqrt{-\lambda})}, \\
& W\left\{\sigma_{-}, \rho\right\}=\frac{-2^{3 / 4}(-\lambda)^{3 / 8} \sqrt{\pi}}{\Gamma\left(\frac{1}{4}-\frac{1}{2 \sqrt{-\lambda}}\right)} .
\end{aligned}
$$

As in Proposition 5.2, $W\left\{\sigma_{-}, \rho\right\}=0$ corresponds to poles of the $\Gamma$-function, i.e.,

$$
\frac{1}{4}-\frac{1}{2 \sqrt{-\lambda_{1}}}=-(n-1), \quad n=1,2, \ldots ; \quad \text { or } \lambda_{1}^{(n)}=-\frac{1}{4(n-3 / 4)^{2}} .
$$

At this value of $\lambda_{1}$, we have

$$
W\left\{\sigma_{+}, \rho\right\}=\frac{(-1)^{n-1} 2^{-1 / 4}\left(-\lambda_{1}\right)^{3 / 8} \sqrt{\pi}}{\Gamma(n-1 / 2)}
$$

since $\cos ((n-3 / 4) \pi)=(-1)^{n} \cos (-3 / 4 \pi)=(-1)^{n-1} \cos (\pi / 4)$. Using the formula for $\partial\left(\Gamma^{-1}\right) / \partial x$ at a pole, we find that

$$
\frac{\partial W}{\partial \lambda}\left(\sigma_{-}, \rho\right)=-2^{7 / 4}\left(-\lambda_{1}\right)^{3 / 8}\left(2 \sqrt{-\lambda_{1}}\right)^{-3} \sqrt{\pi}\left[(-1)^{n}(n-1) !\right]
$$

By (4.30),

$$
\frac{T}{\gamma_{+}^{2}}\left(z_{0}^{-3} \tilde{f}, z_{0}^{-2} \tilde{\lambda}_{1}\right)=\left(-\tilde{\lambda}_{1}\right)^{-1 / 2} z_{0}\left[\frac{8\left(-\tilde{\lambda}_{1}\right)^{3 / 2}}{\tilde{f}}\right]^{2 n-3 / 2} \exp \left(-\frac{4\left(-\tilde{\lambda}_{1}\right)^{3 / 2}}{3 \tilde{f}}\right)
$$

at $\lambda_{1}=z_{0}^{-2} \tilde{\lambda}_{1}=-1 / 4(n-3 / 4)^{2}$. Combining the last three formulae with (5.5) and using $(n-1) ! \Gamma(n-1 / 2)=2^{-(2 n-2)}(2 n-2) ! \sqrt{\pi}, \tilde{\lambda}_{1}=-1 / 4, z_{0}=n-3 / 4$, we obtain (5.7).

§6. The Oppenheimer formula. Our goal in this section is to prove (1.5) and its analogue for excited states. This also proves (1.13) and its analogue for excited states. Because of the connection between the Stark problem and anharmonic oscillators with $\left(m^{2}-1 / 4\right) x^{-2}$ terms (Proposition 1.2$)$, we begin by analyzing 
that problem, proving a formula ((6.1), below) first obtained non-rigorously by Banks, Bender, and Wu [7]. From this result, (1.13) will follow fairly easily. We emphasize that the idea of obtaining the Oppenheimer formula from the Banks-Bender-Wu formula is due independently to Benassi and Grecchi [4].

TheOREM 6.1. Fix $m=0,1,2, \ldots$. Let $\tilde{\Lambda}_{k, m}$ be the analytic continuation of the kth eigenvalue $(k=1,2, \ldots)$ of $\left(-d^{2} / d v^{2}\right)+v^{2}+\left(m^{2}-1 / 4\right) v^{-2}+\beta v^{4}$ $(x \in(-\infty, \infty))$ to the cut plane $\mathrm{C} \backslash(-\infty, 0]$. Then as $\mu \downarrow 0$,

$$
\begin{aligned}
\operatorname{Im} \tilde{\Lambda}_{k, m}(-\mu+i O)= & 2^{4 k+2 m-1}[(k-1) !(k+m-1) !]^{-1} \\
& \times \mu^{-2 k-m-1} \exp (-2 / 3 \mu) \cdot\left[1+O\left(\mu^{\alpha}\right)\right]
\end{aligned}
$$

for some $\alpha>0$.

Remark. Formula (6.1) holds also for non-integral $m$, if $(k+m-1)$ ! is interpreted as $\Gamma(k+m)$. This appears at first sight to contradict (5.1), which after all involves the value $m=1 / 2$. But notice that $\Lambda_{k}=\tilde{\Lambda}_{2 k, m=1 / 2}$. With this realization, and $(k-1) ! \Gamma(k+1 / 2)=2^{-(2 k-1)}(2 k-1) ! \sqrt{\pi}$, one reconciles $(5.1)$ and (6.1).

As in the case " $m=1 / 2$ " discussed in section 5 , this theorem follows from Propositions 1.1 and 2.3 with

Proposition 6.2. Fix $m=0,1,2, \ldots$ Let $H_{m}(\tilde{f}, \tilde{\lambda}, z)$ be the normalized Wronskian for the resonance solution at infinity and the Dirichlet solution at the origin for the Titchmarsh problem,

$$
\left(-\frac{d^{2}}{d x^{2}}-\left((1 / 4) m^{2}-1 / 4\right) x^{-2}-z x^{-1}-\tilde{f} x-\lambda\right) u=0 .
$$

Fix $\tilde{\lambda}_{0}=-1 / 4$ and $z_{0}=k+1 / 2 m-1 / 2$, so that $H_{m}\left(\tilde{f}=0, \tilde{\lambda}_{0}, z_{0}\right)=0$. Then for $\tilde{\lambda}_{0}$ fixed and $\tilde{f}$ near zero and positive, there is a unique solution

$\tilde{z}(f)$ of $H_{m}(\tilde{f}, \tilde{\lambda}, z)=0$ with $\operatorname{Im} \tilde{z}(f)$

$$
\sim-\frac{1}{2}[(k-1) !(k+m-1) !]^{-1} \tilde{f}^{-2 k-m+1} \exp (1 / 6 \tilde{f}) .
$$

Proof. We first consider the case $m \neq 0$. The argument leading to (5.5) did not depend on the value of $g$, so (5.5) is valid here also. Normalizing $\rho$ so that

$$
W\left\{\sigma_{ \pm}, \rho\right\}=\lim _{x \rightarrow 0}\left[x^{-1 / 2+m / 2} \sigma_{ \pm}(x) / \Gamma(m)\right]
$$

we have (by (b.17)) that

$$
W\left\{\sigma_{-}, \rho\right\}=(2 \sqrt{-\lambda})^{1 / 2-m / 2} / \Gamma\left(1 / 2+m / 2-\frac{1}{2 \sqrt{-\lambda}}\right)
$$


and (by (b.18)) that

$$
W\left\{\sigma_{+}, \rho\right\}=\frac{(2 \sqrt{-\lambda})^{1 / 2-m / 2}}{\Gamma\left(1 / 2+m / 2+\frac{1}{2 \sqrt{-\lambda}}\right)}\left\{\frac{q_{m}(\pi(m / 2-1 / 2))}{q_{m}(\pi / 2 \sqrt{-\lambda})}\right\}
$$

with $q_{m}(x)=\cos x$ for $m$ odd and $\sin x$ for $m$ even. By the by now familiar analysis the zeroes of $W\left\{\sigma_{+}, \rho\right\}$ occur at $\lambda_{k}=-1 /(m+2 k-1)^{2}$, at which values

$$
W\left\{\sigma_{+}, \rho\right\}=(-1)^{k}(2 \sqrt{-\lambda})^{1 / 2-m / 2} \Gamma(k+m)
$$

and

$$
\frac{\partial}{\partial \lambda} W\left\{\sigma_{-}, \rho\right\}=(2 \sqrt{-\lambda})^{1 / 2-m / 2}(-1)^{k}(k-1) ! 2(2 \sqrt{-\lambda})^{-3}
$$

Moreover, by (4.30)

$$
\frac{T}{\gamma_{+}^{2}}=\left(-\tilde{\lambda}_{0}\right)^{-1 / 2} z_{0}\left[\frac{8\left(-\tilde{\lambda}_{0}\right)^{3 / 2}}{\tilde{f}}\right]^{m+2 k-1} \exp \left(-4\left(-\tilde{\lambda}_{0}\right)^{3 / 2} / 3 \tilde{f}\right) .
$$

This leads to (6.2) for $m>0$. For $m=0$, a similar argument using (b.17b) and the first equation in (b.21) gives the result.

As a preliminary to the calculation of the width for the Stark problem, we want to compute the first-order shift. To begin with, we compute:

LEMMA 6.3. Uniformly in the cut plane,

$$
\tilde{\Lambda}_{k, m}(\beta)=2 m+4 k-2+\beta B_{k, m}+O\left(\beta^{2}\right),
$$

where

$$
B_{k, m}=\left(m^{2}-3 m+2\right)+6 k^{2}+k(6 m-6) .
$$

Proof. Consider the two-dimensional oscillator

$$
p_{x}^{2}+x^{2}+p_{y}^{2}+y^{2}+\beta\left(x^{2}+y^{2}\right)^{2}=H_{0}+\beta r^{4} .
$$

As usual, we let $a_{x}=2^{-1 / 2}(x+d / d x), a_{x}^{\dagger}=2^{-1 / 2}(x-d / d x), a_{y} a_{y}^{\dagger}$ similarly, and $a_{ \pm}=2^{-1 / 2}\left(a_{x} \pm i a_{y}\right)$. Then $N_{ \pm}=a_{ \pm}^{\dagger} a_{ \pm}$has eigenvalues $n_{ \pm}=0,1,2, \ldots$, and

$$
\begin{aligned}
H_{0} & =2 N_{+}+2 N_{-}+2, \\
L & =N_{+}-N_{-},
\end{aligned}
$$


so that if $\psi_{n_{+}, n_{-}}$is the obvious eigenvector, then (using results of Simon [48] to conclude that perturbation theory is asymptotic in the cut plane):

$$
\begin{gathered}
\tilde{\Lambda}_{k, m}(0)=2 n_{+}+2 n_{-}-2 \\
B_{k, m}=\left(\psi_{n_{+}, n_{-}}, r^{4} \psi_{n_{+}, n_{-}}\right)=\left\|r^{2} \psi_{n_{+}, n_{-}}\right\|^{2},
\end{gathered}
$$

with $n_{ \pm}$related to $k$ and $m$ by $m=n_{+}-n_{-}, k=n_{-}+1$; i.e., $n_{+}=m+k-1$, $n_{-}=k-1$. The claimed formula for $\tilde{\Lambda}_{k, m}(0)$ is now obvious. To get (6.5), we first calculate that

$$
x^{2}+y^{2}=a_{+} a_{-}+a_{-}^{\dagger} a_{+}^{\dagger}+\frac{1}{2} H_{0}
$$

So, by the well-known relation giving the actions of $a_{ \pm}$and $a_{ \pm}^{\dagger}$,

$$
\begin{aligned}
\left(x^{2}+y^{2}\right) \psi_{n_{+}, n_{-}}= & \sqrt{n_{+} n_{-}} \psi_{n_{+}-1, n_{-}-1}+\sqrt{\left(n_{+}+1\right)\left(n_{-}+1\right)} \psi_{n_{+}+1, n_{-}+1} \\
& +\left(n_{+}+n_{-}+1\right) \psi_{n_{+}, n_{-}}
\end{aligned}
$$

Thus, by (6.6),

$$
B_{k, m}=n_{+} n_{-}+\left(n_{+}+1\right)\left(n_{-}+1\right)+\left[n_{+}+n_{-}+1\right]^{2} .
$$

which yields (6.5).

LEMMA 6.4. Let $E_{k, q, m}(F)$ be the solution (unique for $F$ small and positive) of

$$
\tilde{\Lambda}_{k, m}\left(-E, \frac{F}{2}\right)+\tilde{\Lambda}_{q, m}\left(-E,-\frac{F}{2}-i O\right)=2
$$

where

$$
\tilde{\Lambda}_{k, m}(\alpha, \beta)=\alpha^{1 / 2} \tilde{\Lambda}_{k, m}\left(\beta \alpha^{-3 / 2}\right)
$$

Then

$$
E_{k, q, m}(F)=\frac{1}{4} N^{-2}+3(k-q) N F+O\left(F^{2}\right)
$$

where

$$
N=m+k+q-1
$$

Remark. By scaling, $\tilde{\Lambda}_{k, m}(\alpha, \beta)$ is the $m$ th eigenvalue of $\left(-d^{2} / d v^{2}\right)+d v^{2}+$ $\beta v^{4}+\left(m^{2}-\frac{1}{4}\right) v^{-2}$. Thus, by Propositions 1.2 and $2.4 E_{k, q, m}$ is a resonance eigenvalue of the Stark Hamiltonian $-\Delta-1 / r-F z$. 
Proof. Consider $F=0$. Then (6.7) becomes:

$$
2=\sqrt{-E}(4 m+4 k+4 q-4)=\sqrt{-E}(4 N)
$$

by (6.4). From this the formula for $E(0)$ is obvious. For non-zero $F$, (6.7) becomes

$$
\begin{aligned}
2 & =\sqrt{-E}\left[(4 N)+\frac{F}{2}(-E)^{-3 / 2}\left(B_{k, m}-B_{q, m}\right)+O\left(F^{2}\right)\right] \\
& =\sqrt{-E}\left[4 N+\frac{F}{2}(2 N)^{3}\left(B_{k, m}-B_{q, m}\right)+O\left(F^{2}\right)\right]
\end{aligned}
$$

so

$$
\begin{aligned}
\sqrt{-E} & =\left[2 N+\frac{F}{4}(2 N)^{3}\left(B_{k, m}-B_{q, m}\right)+O(F)^{2}\right]^{-1} \\
& =(2 N)^{-1}-\frac{F}{4}(2 N)\left(B_{k, m}-B_{q, m}\right)+O\left(F^{2}\right) .
\end{aligned}
$$

Squaring, we find that

$$
E=-(2 N)^{-2}+\frac{1}{2}\left(B_{k, m}-B_{q, m}\right) F,
$$

so that (6.8a) follows from (6.5).

We can now state the main result on the Stark width (this agrees with non-rigorous calculations in $[4,12,46]$.)

THEOREM 6.5. Let $E_{k, q, m}(F)$ be the Stark resonance energy solving (6.7). Then

$$
\begin{aligned}
\operatorname{Im} E_{k, q, m}(F)= & -\frac{1}{4}[(q-1) !(q+m-1) !]^{-1} e^{3(k-q)} F^{-2 q-m+1} N^{-6 q-3 m} \\
& \times \exp \left(-1 / 6 N^{3} F\right) \cdot\left(1+O\left(F^{\alpha}\right)\right)
\end{aligned}
$$

where $N=k+q+m-1$. In particular, (1.5) holds for $-2 \operatorname{Im} E_{1,1,0}$.

Proof. By Theorem 2.5,

$$
\operatorname{Im} E_{k, q, m}(F)=-C \operatorname{Im}\left[\tilde{\Lambda}_{q, m}\left(-E_{k, q, m}(0),-\frac{F}{2}-i O\right)\right]\left(1+O\left(F^{\alpha}\right)\right),
$$

with $C=\left.e^{\gamma}\left[4 N \partial(-E)^{1 / 2} / \partial E\right]^{-1}\right|_{E(0)}=e^{\gamma}(2 N)^{-2}$ and $\gamma=$ the first-order term in the exponential. Thus writing

$$
\begin{gathered}
E_{k, q, m}(F)=-\frac{1}{4} N^{-2}+A_{k, q, m} F, \text { we have that } \\
\gamma=\left(-\frac{4}{3}\right)\left(\frac{3}{2}(2 N)^{-1} A_{k, q, m}\right)=3(k-q)
\end{gathered}
$$

by (6.8). Using (6.1) we obtain (6.9). 
Finally, we obtain the asymptotics of the perturbation coefficients in the Stark problem using the general analogue of $(1.12)$ for non-even $\Gamma$ 's $[26,4,46]$,

$A_{n}=(\pi)^{-1} \int_{0}^{R} F^{-n-1}\left[\operatorname{Im} E(F+i O)+(-1)^{n} \operatorname{Im} E(-F+i O)\right] d F+O\left(R^{-2 n}\right)$.

Since $E_{k, q, m}(-F+i O)=E_{q, k, m}(+F+i O)$ and since for $q>k$, (respectively $k>q) E_{k, q, m}(F)$, dominates (respectively is dominated by) $E_{q, k, m}(F)$ for $F$ small, we obtain:

THEOREM 6.6. Let $A_{n}^{k, q, m}$ denote the $n$th Rayleigh-Schrödinger coefficient for $E_{k, q, m}$. Then to order $\left(1+O\left(n^{-a}\right)\right)$,

$$
\begin{aligned}
A_{n} \sim & -(4 \pi)^{-1}[(q-1) !(q+m-1) !]^{-1} e^{3(k-q)} N^{-3} 6^{2 q+m-1}\left(6 N^{3}\right)^{n} \\
& \times(n+2 q+m-2) !, \quad \text { if } q>k ; \\
\sim & -(4 \pi)^{-1}(-1)^{n}[(k-1) !(k+m-1) !]^{-1} e^{3(q-k)} N^{-3} 6^{2 k+m-1}\left(6 N^{3}\right)^{n} \\
& \times(n+2 k+m-2) !, \quad \text { if } k>q ; \\
\sim & -(4 \pi)^{-1}\left[1+(-1)^{n}\right][(k-1) !(k+m-1) !]^{-1} N^{-3} 6^{2 k+m-1}\left(6 N^{3}\right)^{n} \\
& \times(n+2 k+m-2) !, \quad \text { if } k=q .
\end{aligned}
$$

In particular, (1.13) holds.

Appendix A. Calculation of the tunneling factor. We calculate the tunneling factor of section 4 ,

$$
T(f, \lambda) \equiv \exp \left(-2 \int_{t_{1}}^{t_{2}}(C(x))^{1 / 2} d x\right),
$$

where $C(x)=g x^{-2}-x^{-1}-f x-\lambda$, and the turning points $t_{1}$ and $t_{2}$ are the roots of $C=0$ respectively near $-(1+\sqrt{1-4 \lambda g}) / 2 \lambda$ and $-\lambda / f+1 / \lambda$. For the purposes of this calculation, define the approximate turning points

$$
\begin{aligned}
& t_{2}^{\prime} \equiv-\lambda\left(1+\sqrt{1-4 f / \lambda^{2}}\right) / 2 f \approx t_{2} \\
& t_{1}^{\prime} \equiv-\lambda\left(1-\sqrt{1-4 f / \lambda^{2}}\right) \\
& t_{1}^{\prime \prime} \equiv(-1 / 2 \lambda)(1+\sqrt{1+4 g \lambda}) .
\end{aligned}
$$


$t_{1}^{\prime}$ would be the lower turning point if $g x^{-2}$ were neglected, and $t_{1}^{\prime \prime}$ would be the lower turning point if $f x$ were neglected. Then if $g \geqslant 0$,

$$
\begin{aligned}
& \int_{t_{1}}^{t_{2}}(C(x))^{1 / 2} d x=\int_{t_{1}^{\prime}}^{t_{2}^{\prime}}(-\lambda-f x-1 / x)^{1 / 2} d x+\int_{t_{1}^{\prime \prime}}^{f^{-\alpha}}\left(-\lambda-1 / x+g / x^{2}\right)^{1 / 2} d x \\
& -\int_{t_{1}^{\prime}}^{f^{-\alpha}}(-\lambda-1 / x)^{1 / 2} d x+O\left(f^{\alpha}\right)+O\left(f^{1-2 \alpha}\right) \\
& =\frac{2}{3} \sqrt{f t_{2}^{\prime}}\left[\left(t_{2}^{\prime}+t_{1}^{\prime}\right) E\left(\frac{\pi}{2}, \sqrt{1-t_{1}^{\prime} / t_{2}^{\prime}}\right)-2 t_{1}^{\prime} F\left(\frac{\pi}{2}, \sqrt{1-t_{1}^{\prime} / t_{2}^{\prime}}\right)\right] \\
& +\left.\sqrt{g-x-\lambda x^{2}}\right|_{t_{1}^{\prime \prime}} ^{f^{-\alpha}}-\left.\sqrt{g} \ln \left(\frac{2 g-x+2 \sqrt{g} \sqrt{g-x-\lambda x^{2}}}{x}\right)\right|_{t_{1}^{\prime \prime}} ^{f^{-\alpha}} \\
& -\left(\frac{1}{2 \sqrt{-\lambda}}\right) \ln \left(\frac{2 \sqrt{-\lambda} \sqrt{-\lambda f^{-2 \alpha}-f^{-\alpha}+g}-2 \lambda f^{-\alpha}-1}{-2 \lambda t_{1}^{\prime \prime}-1}\right) \\
& -\left.\sqrt{-\lambda x^{2}-x}\right|_{t_{1}^{\prime}} ^{f^{-\alpha}}+\left(\frac{1}{2 \sqrt{-\lambda}}\right) \ln \left(\frac{2 \sqrt{-\lambda} \sqrt{-\lambda f^{-2 \alpha}-f^{-\alpha}}-2 \lambda f^{-\alpha}-1}{-2 \lambda t_{1}^{\prime}-1}\right) \\
& +O\left(f^{+\alpha}+f^{1-2 \alpha}\right) \\
& =\frac{2}{3} \sqrt{-\lambda+f / \lambda}\left[\frac{-\lambda}{f}\left[1+\frac{1}{2}\left(\frac{t_{1}^{\prime}}{t_{2}^{\prime}}\right)\left(\ln \left(4 \sqrt{\frac{t_{2}^{\prime}}{t_{1}^{\prime}}}\right)-\frac{1}{2}\right)\right)-2 t_{1}^{\prime} \ln \left(4 \sqrt{\frac{t_{2}^{\prime}}{t_{1}^{\prime}}}\right)\right] \\
& -\sqrt{g} \ln \left(\frac{(2 \sqrt{-g \lambda}-1) t_{1}^{\prime \prime}}{2 g-t_{1}^{\prime \prime}}\right)+\left(\frac{1}{2 \sqrt{-\lambda}}\right) \ln \left(\frac{-2 \lambda t_{1}^{\prime \prime}-1}{-2 \lambda t_{1}^{\prime}-1}\right) \\
& +O\left(f^{\alpha}+f^{1-2 \alpha}\right) \\
& =\frac{2}{3} \frac{(-\lambda)^{3 / 2}}{f}-\frac{1}{\sqrt{-\lambda}} \ln (-4 \lambda / \sqrt{f})-\left(\frac{1}{2 \sqrt{-\lambda}}\right) \\
& -\sqrt{g} \ln \left[\frac{(2 \sqrt{-g \lambda}-1)(1+\sqrt{1+4 g \lambda})}{-4 g \lambda-1-\sqrt{1+4 g \lambda}}\right]+\left(\frac{1}{2 \sqrt{-\lambda}}\right) \ln (\sqrt{1+4 g \lambda}) \\
& +O\left(f^{\alpha}+f^{1-2 \alpha}\right)
\end{aligned}
$$


This equation uses some tabulated integrals [17] and facts about the elliptic integrals, which we have collected in Appendix B. Thus

$$
\begin{aligned}
T(f, \lambda)= & {\left[\frac{16 \lambda^{2} e}{f \sqrt{1+4 g \lambda}}\right]^{1 / \sqrt{-\lambda}}\left[\frac{(2 \sqrt{-\lambda g}-1)(1+\sqrt{1+4 g \lambda})}{-4 g \lambda-1-\sqrt{1+4 g \lambda}}\right]^{2 \sqrt{g}} } \\
& \times \exp \left(-\left(\frac{4}{3}\right)(-\lambda)^{3 / 2} / f\right) \cdot\left(1+O\left(f^{\alpha}+f^{1-2 \alpha}\right)\right) .
\end{aligned}
$$

If $-1 / 4 \leqslant g<0$, then the integral from $t^{\prime \prime}$ to $f^{-\alpha}$ has a different form, and

$$
\begin{aligned}
T(f, \lambda)= & {\left[\frac{16 \lambda^{2} e}{f \sqrt{1+4 g \lambda}}\right]^{1 / \sqrt{-\lambda}} \exp ((\pi+2 \arcsin (1 / \sqrt{1+4 \lambda g}) / \sqrt{-g})) } \\
& \times \exp \left(-\frac{4}{3}(-\lambda)^{3 / 2} / f\right) \cdot\left(1+O\left(f^{\alpha}+f^{1-2 \alpha}\right)\right) .
\end{aligned}
$$

Appendix B. Some properties of special functions. The following facts are compiled from $[1,9,17]$.

1. Airy functions. The functions $\operatorname{Ai}(z), B i(z)$, and $\operatorname{Ai}(z \exp ( \pm 2 \pi i / 3))$ solve

$$
w^{\prime \prime}-z w=0 \text {. }
$$

Any two of them are linearly independent, $A i(z)$ and $B i(z)$ are real for real $z$, and they are related by analytic continuation and the formula

$$
B i(z)=\exp (\pi i / 6) A i(z \exp (2 \pi i / 3))+\exp (-\pi i / 6) A i(z \exp (-2 \pi i / 3)) \text {. }
$$

Some of their Wronskians are:

$$
\begin{aligned}
W\{\operatorname{Ai}(z), B i(z)\} & =1 / \pi \\
W\{\operatorname{Ai}(z), \operatorname{Ai}(z \exp ( \pm 2 \pi i / 3))\} & =\exp (\mp \pi i / 6) / 2 \pi \\
W\{\operatorname{Bi}(z), \operatorname{Ai}(z \exp ( \pm 2 \pi i / 3))\} & =\mp i \exp (\mp \pi i / 6) / 2 \pi
\end{aligned}
$$

For small $z$,

$$
\begin{aligned}
& A i(z)=3^{-2 / 3} / \Gamma(2 / 3)-3^{-1 / 3} z / \Gamma(1 / 3)+\cdots \\
& B i(z)=3^{-1 / 6} / \Gamma(2 / 3)+3^{1 / 6} z / \Gamma(1 / 3)+\cdots
\end{aligned}
$$

For large $z,|\arg z|<\pi$,

$$
\operatorname{Ai}(z)=\frac{1}{2 \sqrt{\pi}} z^{-1 / 4} \exp \left(-2 z^{3 / 2} / 3\right) \cdot\left(1+O\left(z^{-3 / 2}\right)\right)
$$


and for large $z,|\arg z|<\pi / 3$,

$$
B i(z)=\frac{1}{\sqrt{\pi}} z^{-1 / 4} \exp \left(+2 z^{3 / 2} / 3\right) \cdot\left(1+O\left(z^{-3 / 2}\right)\right)
$$

Equations (b.5) and (b.6) may also be differentiated. Moreover, if $z>0$, then $d A i(z) / d z<0$ and $d B i(z) / d z>0$.

The Airy functions we use are of the form

$$
\begin{aligned}
& a(x ; f, \lambda) \equiv A i\left(-f^{1 / 3}(x+\lambda / f)\right), \\
& b(x ; f, \lambda) \equiv B i\left(-f^{1 / 3}(x+\lambda / f)\right),
\end{aligned}
$$

and

$$
c(x ; f, \lambda) \equiv A i\left(-f^{1 / 3}(x+\lambda / f) \exp (2 \pi i / 3)\right)
$$

Therefore

$$
\begin{aligned}
& W\{a, b\}=-f^{1 / 3} / \pi \\
& W\{a, c\}=-f^{1 / 3} \exp (-\pi i / 6) / 2 \pi,
\end{aligned}
$$

and

$$
W\{b, c\}=f^{1 / 3} \exp (\pi i / 3) / 2 \pi
$$

If $x<\lambda / f-f^{-1 / 3}$, then

$$
a(x ; f, \lambda)=\frac{f^{1 / 6}}{2 \sqrt{\pi}}(-\lambda-f x)^{-1 / 4} \exp \left(-\frac{2}{3}(-\lambda-f x)^{3 / 2} / f\right) \cdot\left(1+O\left(f^{1 / 2}\right)\right),
$$

and

$$
b(x ; f, \lambda)=\frac{f^{1 / 6}}{2 \sqrt{\pi}}(-\lambda-f x)^{-1 / 4} \exp \left(+\frac{2}{3}(-\lambda-f x)^{3 / 2} / f\right) \cdot\left(1+O\left(f^{1 / 2}\right)\right) .
$$

If $x=-\lambda / f+r \exp (\pi i / 3)$ and $r$ is real and $>f^{-1 / 3}$, then

$$
a(x ; f, \lambda)=\exp (+\pi i / 6) \frac{f^{-1 / 12}}{2 \sqrt{\pi}} r^{-1 / 4} \exp \left(+\frac{2}{3} f^{1 / 2} r^{3 / 2}\right) \cdot\left(1+O\left(f^{1 / 2}\right)\right),
$$


and

$$
c(x ; f, \lambda)=\frac{f^{-1 / 12}}{2 \sqrt{\pi}} r^{-1 / 4} \exp \left(-\frac{2}{3} f^{1 / 2} r^{3 / 2}\right) \cdot\left(1+O\left(f^{1 / 2}\right)\right) .
$$

2. Whittaker functions. The functions

$$
\begin{aligned}
& W_{1 / 2 \sqrt{-\lambda}, \sqrt{g+1 / 4}}(2 \sqrt{-\lambda} x), \\
& W_{-1 / 2 \sqrt{-\lambda}, \sqrt{g+1 / 4}}(2 \sqrt{-\lambda} x \exp (\pi i)),
\end{aligned}
$$

and

$$
W_{-1 / 2 \sqrt{-\lambda}, \sqrt{g+1 / 4}}(2 \sqrt{-\lambda} x \exp (-\pi i))
$$

solve the equation

$$
\left(-d^{2} / d x^{2}+g / x^{2}-1 / x-\lambda\right) w(x)=0 .
$$

The Wronskian is:

$$
\begin{aligned}
& W\left\{W_{1 / 2 \sqrt{-\lambda}} \sqrt{g+1 / 4}(2 \sqrt{-\lambda} x), W_{-1 / 2 \sqrt{-\lambda}} \sqrt{g+1 / 4}(\exp ( \pm \pi i) 2 \sqrt{-\lambda} x)\right\} \\
& =2 \sqrt{-\lambda} \exp (\mp \pi i / 2 \sqrt{-\lambda}) \text {. }
\end{aligned}
$$

For small $z$,

$$
W_{\kappa, 0}(z)=-z^{1 / 2} \ln (z) / \Gamma(1 / 2-\kappa)+O\left(z^{1 / 2}\right),
$$

and for $g>-1 / 4$,

$$
\begin{aligned}
W_{\kappa, \sqrt{g+1 / 4}}(z)= & (2 \sqrt{g+1 / 4}) z^{1 / 2-\sqrt{g+1 / 4}} / \Gamma(1 / 2+\sqrt{g+1 / 4}-\kappa) \\
& +O\left(z^{3 / 2-\sqrt{g+1 / 4}}+z^{1 / 2+\sqrt{g+1 / 4}} \ln (z)\right) .
\end{aligned}
$$

For the Neumann case with $g=-3 / 16$, we need somewhat more detailed information, and (b.17) taken to higher order in $z$ is

$$
\begin{aligned}
W_{\kappa, 1 / 4}(z)= & \sqrt{\pi} z^{1 / 4} / \Gamma(3 / 4-\kappa)-\sqrt{\pi} z^{3 / 4} / \Gamma(1 / 2-\kappa) \\
& -2 \kappa \sqrt{\pi} z^{5 / 4} / \Gamma(3 / 4-\kappa)+\cdots .
\end{aligned}
$$

(The logarithmic terms of (b.17) occur only when the second subscript is $n / 2, n$ integral.) For large $z,|\arg z| \leqslant 3 \pi / 2-\delta, \delta>0$,

$$
W_{\kappa, \sqrt{g+1 / 4}}(z)=z^{\kappa} \exp (-z / 2) \cdot(1+O(1 / z)) .
$$


The two functions

$$
W_{1 / 2 \sqrt{-\lambda}, \sqrt{g+1 / 4}}(2 \sqrt{-\lambda} x)
$$

and

$$
\sigma_{+}(x)=\left\{\begin{array}{cl}
\frac{1}{2 \cos (\pi / 2 \sqrt{-\lambda})}\left\{W_{-1 / 2 \sqrt{-\lambda}, \sqrt{g+1 / 4}}\left(e^{i \pi} 2 \sqrt{-\lambda} x\right)\right. & \\
\left.+W_{-1 / 2 \sqrt{-\lambda}}, \sqrt{g+1 / 4}\left(e^{-i \pi} 2 \sqrt{-\lambda x}\right)\right\}, & g \neq n^{2}-1 / 4 \\
\frac{-1}{2 i \sin (\pi / 2 \sqrt{-\lambda})}\left\{W_{-1 / 2 \sqrt{-\lambda}}, \sqrt{g+1 / 4}\left(e^{i \pi} 2 \sqrt{-\lambda} x\right)\right. & \\
\left.-W_{-1 / 2 \sqrt{-\lambda}}, \sqrt{g+1 / 4}\left(e^{-i \pi} 2 \sqrt{-\lambda} x\right)\right\}, & g=n^{2}-1 / 4,
\end{array}\right.
$$

$n=0,1, \ldots$, are real for real $x$, and their Wronskian is

$$
W\left\{W_{1 / 2 \sqrt{-\lambda}} \sqrt{g+1 / 4}(2 \sqrt{-\lambda} x), \sigma_{+}(x)\right\}=2 \sqrt{-\lambda} .
$$

The function (b.19a) has the proper behavior for large $x$ and, at the exceptional values $1 / 2 \sqrt{-\lambda}=1 / 2+\sqrt{g+1 / 4}+k, k=0,1,2, \ldots$, also for $x \rightarrow 0$. The function (b.19b) has the improper behavior in both limits. The double definition ensures this even when $1 / 2 \sqrt{-\lambda}$ has an exceptional value and $g=n^{2}-1 / 4$ (at which $\cos (\pi / 2 \sqrt{-\lambda})$ would be zero). For small $x$,

$$
\sigma_{+}(x) \sim\left\{\begin{array}{l}
\frac{(2 \sqrt{-\lambda} x)^{1 / 2}}{\Gamma\left(\frac{1}{2}+\frac{1}{2 \sqrt{-\lambda}}\right) \sin \left(\frac{\pi}{2 \sqrt{-\lambda}}\right)} \ln (2 \sqrt{-\lambda} x), \\
g=-1 / 4 ; \\
\frac{\Gamma(2 \sqrt{g+1 / 4}) \cos \left(\pi\left(\frac{1}{2}-\sqrt{g+1 / 4}\right)\right)}{\Gamma\left(\frac{1}{2}+\sqrt{g+1 / 4}+\frac{1}{2 \sqrt{-\lambda}}\right) \cos \left(\frac{\pi}{2 \sqrt{-\lambda}}\right)}(2 \sqrt{-\lambda} x)^{1 / 2-\sqrt{g+1 / 4}} \\
g \neq n^{2}-1 / 4, g>1 / 4 ; \\
\frac{\Gamma(2 \sqrt{g+1 / 4}) \sin \left(\pi\left(\frac{1}{2}-\sqrt{g+1 / 4}\right)\right)}{\Gamma\left(\frac{1}{2}+\sqrt{g+1 / 4}+\frac{1}{2 \sqrt{-\lambda}}\right) \sin \left(\frac{\pi}{2 \sqrt{-\lambda}}\right)}(2 \sqrt{-\lambda} x)^{1 / 2-\sqrt{g+1 / 4}} \\
g=n^{2}-1 / 4, n=1,2, \ldots
\end{array},\right.
$$


For large, real $z$,

$$
\sigma_{+}(x)=(2 \sqrt{-\lambda} x)^{-1 / 2 \sqrt{-\lambda}} \exp (\sqrt{-\lambda} x) \cdot(1+0(1 / x))
$$

3. Some integrals and approximations. If $a>b>0$, then

$$
\begin{gathered}
\int_{b}^{a} \sqrt{(a-x)(x-b) / x} d x=\frac{2}{3} \sqrt{a}[(a+b) E(\pi / 2, \sqrt{(a-b) / a}) \\
-2 b F(\pi / 2, \sqrt{(a-b) / a})] .
\end{gathered}
$$

Define $k^{\prime}=\sqrt{1-k}$. Then series expansions for the complete elliptic integrals in two different limits are

$$
\begin{aligned}
E(\pi / 2, k) \equiv & E(k)=\frac{\pi}{2} F\left(-1 / 2,1 / 2 ; 1 ; k^{2}\right) \\
= & \frac{\pi}{2}\left\{1-k^{2} / 2-1^{2} \cdot 3 k^{4} / 2^{2} \cdot 4-\cdots\right\} \\
= & 1+\left(\ln \left(4 / k^{\prime}\right)-1 / 1 \cdot 2\right)\left(k^{\prime}\right)^{2} / 2+1^{2} \cdot 3\left(\ln \left(4 / k^{\prime}\right)\right. \\
& -2 / 1 \cdot 2-1 / 3 \cdot 4)\left(k^{\prime}\right)^{4} / 2^{2} \cdot 4+\cdots
\end{aligned}
$$

and

$$
\begin{aligned}
F(\pi / 2, k) \equiv K(k) & =\frac{\pi}{2} F\left(1 / 2,1 / 2 ; 1 ; k^{2}\right) \\
& =\frac{\pi}{2}\left\{1+(1 / 2)^{2} k^{2}+(1 \cdot 3 / 2 \cdot 4)^{2} k^{4}+\cdots\right\} \\
& =\ln \left(4 / k^{\prime}\right)+(1 / 2)^{2}\left(\ln \left(4 / k^{\prime}\right)-2 / 1 \cdot 2\right)\left(k^{\prime}\right)^{2}+\cdots
\end{aligned}
$$

If the integral of (b.23) is incomplete, from $u>b$ to $a$, then

$$
\begin{aligned}
\int_{u}^{a} \sqrt{(a-x)(x-b) / x} d x= & \frac{2}{3} \sqrt{a}[(a+b) E(\arcsin \sqrt{(a-u) /(a-b)}, \sqrt{(a-b) / a}) \\
& -2 b F(\arcsin \sqrt{(a-u) /(a-b)}, \sqrt{(a-b) / a})] \\
& +\frac{2}{3}(u-a-b) \sqrt{(a-u)(u-b) / u}
\end{aligned}
$$


If $k$ is near 1 and $\phi$ is small, then

$$
\begin{aligned}
E(\phi, k) & =\frac{2}{\pi} E\left(k^{\prime}\right) \ln \tan (\phi / 2+\pi / 4)+\frac{\tan \phi}{\cos \phi}\left(\frac{2}{\pi} E\left(k^{\prime}\right)-1-\cdots\right) \\
& =2 \phi+\cdots
\end{aligned}
$$

and

$$
\begin{aligned}
F(\phi, k) & =\frac{2}{\pi} K\left(k^{\prime}\right) \ln \tan (\phi / 2+\pi / 4)-\frac{\tan \phi}{\cos \phi}\left(\frac{2}{\pi} K\left(k^{\prime}\right)-1+\cdots\right) \\
& =\phi+\cdots .
\end{aligned}
$$

Other integrals we use are:

$$
\begin{gathered}
\int \begin{array}{c}
x\left(\sqrt{a+b x+c x^{2}} / x\right) d x= \\
+\frac{b}{2} \int^{x} \frac{d x}{\sqrt{a+b x+c x^{2}}}, \\
\int \frac{d x}{x \sqrt{a+b x+c x^{2}}}+a \int^{x} \frac{d x}{x \sqrt{a+b x+c x^{2}}} \\
-2 \sqrt{b x+c x^{2}} / b x, \quad a=0, b \neq 0 ; \\
\frac{1}{\sqrt{-a}} \arcsin \left((2 a+b x) / x \sqrt{b^{2}-4 a c}\right), \\
-\frac{1}{\sqrt{a}} \ln \left(\frac{2 a+b x+2 \sqrt{a} \sqrt{a+b x+c x^{2}}}{x}\right), \quad a>0 \\
a<0, b^{2}>4 a c ;
\end{array}
\end{gathered}
$$

and

$$
\int^{x} \frac{d x}{\sqrt{a+b x+c x^{2}}}=\frac{1}{\sqrt{c}} \ln \left(2 \sqrt{c} \sqrt{a+b x+c x^{2}}+2 c x+b\right), \quad c>0 .
$$

Appendix C. Construction of the comparison functions. We wish to solve for the coefficients in the expressions

$$
\begin{aligned}
& \Phi_{+}= \begin{cases}\alpha_{+} \sigma_{+}(x)+\beta_{+} \sigma_{-}(x), & \text { Region I; } \\
\gamma_{+} \chi_{+}(x)+\delta_{+} \chi_{-}(x), & \text { Region II; } \\
\epsilon_{+} a(x)+\zeta_{+} b(x), & \text { Region III; }\end{cases} \\
& \eta_{+}= \begin{cases}\epsilon_{+} a(x)+\zeta_{+} b(x), & \text { Region IV; } \\
\theta_{+} \chi_{+}(x)+\iota_{+} \chi_{-}(x), & \text { Region V }\end{cases}
\end{aligned}
$$


By the definition in section $4, \alpha_{+}=1$ and $\beta_{+}=0$. Thus $\Phi_{+}(x)$ is known throughout Region I, and the coefficients in the other regions will be determined by matching at the boundaries. The boundary between Region I and II is at $x=f^{-\alpha}$, at which

$$
\begin{aligned}
& \phi_{+}\left(f^{-\alpha}\right)=\gamma_{+} \chi_{+}\left(f^{-\alpha}\right)+\delta_{+} \chi_{-}\left(f^{-\alpha}\right), \\
& \phi_{+}^{\prime}\left(f^{-\alpha}\right)=\gamma_{+} \chi_{+}^{\prime}\left(f^{-\alpha}\right)+\delta_{+} \chi_{-}^{\prime}\left(f^{-\alpha}\right),
\end{aligned}
$$

and we readily find that

$$
\gamma_{+}=W\left\{\phi_{+}, \chi_{-}\right\} /\left.W\left\{\chi_{+}, \chi_{-}\right\}\right|_{x=f^{-\alpha}}
$$

and

$$
\delta_{+}=W\left\{\phi_{+}, \chi_{+}\right\} /\left.W\left\{\chi_{-}, \chi_{+}\right\}\right|_{x=f^{-\alpha}} .
$$

Since $\phi_{+}$is now known in Region II, we calculate at the next boundary:

$$
\epsilon_{+}=W\left\{\phi_{+}, b\right\} /\left.W\{a, b\}\right|_{x=-\lambda / f-f^{-\beta}}
$$

and

$$
\zeta_{+}=W\left\{\phi_{+}, a\right\} /\left.W\{b, a\}\right|_{x=-\lambda / f-f^{-\beta}}
$$

and so on:

$$
\begin{aligned}
& \boldsymbol{\theta}_{+}=W\left\{\eta_{+}, \chi_{-}\right\} /\left.W\left\{\chi_{+}, \chi_{-}\right\}\right|_{x=-\lambda / f+e^{-\pi i / 3} f^{-\beta}}, \\
& \iota_{+}=W\left\{\eta_{+}, \chi_{+}\right\} /\left.W\left\{\chi_{-}, \chi_{+}\right\}\right|_{x=-\lambda / f+e^{-\pi i / 3} f^{-\beta}}
\end{aligned}
$$

The formulae of Appendix B allow us to obtain useful approximations for these coefficients, the most important of which is $\gamma_{+}$(cf. (4.9) and (4.10)). From (c.2),

$$
\begin{aligned}
\gamma_{+}= & -\frac{1}{2}(2 \sqrt{-\lambda})^{-1 / 2 \sqrt{-\lambda}} \exp (\sqrt{-\lambda} x) C(x)^{-1 / 4} \exp \left(-\int_{t_{1}}^{x} C\left(x^{\prime}\right)^{1 / 2} d x^{\prime}\right) \\
& \left.\cdot\left\{-C^{\prime}(x) / 4 C(x)-C(x)^{1 / 2}+1 / 2 \sqrt{-\lambda} x-\sqrt{-\lambda}\right\}\right|_{x=f^{-\alpha}} \\
= & \frac{1}{2}\left(2 \sqrt{-\lambda} f^{-\alpha}\right)^{-1 / 2 \sqrt{-\lambda}}(-\lambda)^{-1 / 4} \exp \left(\sqrt{-\lambda} f^{-\alpha}\right. \\
& \left.-\int_{t_{1}^{\prime \prime}}^{f^{-\alpha}}\left(-\lambda+g / x^{2}-1 / x\right)^{1 / 2} d x+O\left(f^{1-2 \alpha}\right)\right) \cdot\left\{2 \sqrt{-\lambda}+O\left(f^{\alpha}\right)\right\},
\end{aligned}
$$


with $t_{1}^{\prime \prime}$ from (a.1). Thus

$$
\begin{aligned}
\gamma_{+}= & (-\lambda)^{1 / 4}\left(2 \sqrt{-\lambda} f^{-\alpha}\right)^{-1 / 2 \sqrt{-\lambda}} \exp \left(\sqrt{-\lambda} f^{-\alpha}-\left(-\lambda f^{-2 \alpha}+g-f^{-\alpha}\right)^{1 / 2}\right. \\
+ & \left.\sqrt{g} \ln \left(\left(2 g-x+2 \sqrt{g} \sqrt{g-x-\lambda x^{2}}\right) / x\right)\right|_{t_{1}^{\prime \prime}} ^{f^{-\alpha}} \\
+ & \left.\left.\frac{1}{2 \sqrt{-\lambda}} \ln \left(2 \sqrt{-\lambda} \sqrt{g-x-\lambda x^{2}}-2 \lambda x-1\right)\right|_{t_{1}^{\prime \prime}} ^{f^{-\alpha}}\right) \\
& \cdot\left(1+O\left(f^{\alpha}\right)+O\left(f^{1-2 \alpha}\right)\right), \text { for } g \geqslant 0 \text { by }(\mathrm{b} .28)-(\mathrm{b} .30), \text { which } \\
= & (-\lambda)^{1 / 4}\left[\frac{2 \sqrt{-\lambda} e}{-2 \lambda t_{1}^{\prime \prime}-1}\right]^{1 / 2 \sqrt{-\lambda}}\left[\frac{(2 \sqrt{-g \lambda}-1) t_{1}^{\prime \prime}}{2 g-t_{1}^{\prime \prime}}\right]^{\sqrt{g}} \cdot\left(1+O\left(f^{\alpha}+f^{1-2 \alpha}\right)\right) \\
= & (-\lambda)^{1 / 4}\left[\frac{2 \sqrt{-\lambda} e}{\sqrt{1+4 g \lambda}}\right]^{1 / 2 \sqrt{-\lambda}}\left[\frac{1-2 \sqrt{-g \lambda}}{\sqrt{1+4 g \lambda}}\right]^{\sqrt{g}} \cdot\left(1+O\left(f^{\alpha}+f^{1-2 \alpha}\right)\right) .
\end{aligned}
$$

If $-1 / 4<g<0$, then $\gamma_{+}$becomes $(-\lambda)^{1 / 4}[(2 \sqrt{-\lambda} e / \sqrt{1+4 g \lambda})]^{1 / 2 \sqrt{-\lambda}}$. $\exp ((\pi / 2+\arcsin (1 / \sqrt{1+4 g \lambda})) / \sqrt{-g})$ by (b.29). On the other hand,

$$
\begin{aligned}
\delta_{+}= & \frac{1}{2}(2 \sqrt{-\lambda} x)^{-1 / 2 \sqrt{-\lambda}} \exp (\sqrt{-\lambda} x) C(x)^{-1 / 4} \exp \left(+\int_{t_{1}}^{x} C\left(x^{\prime}\right)^{1 / 2} d x^{\prime}\right) \\
& \left.\cdot\left\{-C^{\prime}(x) / 4 C(x)+C(x)^{1 / 2}+1 / 2 \sqrt{-\lambda} x-\sqrt{-\lambda}\right\}\right|_{x=f^{-\alpha}}
\end{aligned}
$$

Thus

$$
\delta_{+} / \gamma_{+} \exp \left(2 \int_{t_{1}}^{f^{-\alpha}} C(x)^{1 / 2} d x\right)=O\left(f^{\alpha}\right)
$$

and since $\chi_{+} / \chi_{-}=\exp \left(2 \int_{t}^{x}\left(C\left(x^{\prime}\right)\right)^{\frac{1}{2}} d x^{\prime}\right) \geqslant \exp \left(2 \int_{t_{1}}^{f^{-\alpha}}\left(C\left(x^{\prime}\right)\right)^{\frac{1}{2}} d x^{\prime}\right)$ on Region II, $\delta_{+} \chi_{-}(x) / \gamma_{+} \chi_{+}(x)=O\left(f^{\alpha}\right)$ uniformly on Region II, from which it follows that $\phi_{+}(x) / \gamma_{+} \chi_{+}(x)=1+O\left(f^{\alpha}+f^{1-2 \alpha}\right)$ as in (4.11b). At the next boundary, $x=-\lambda / f-f^{-\beta}$,

$$
\begin{aligned}
& \phi_{+}(x) / \gamma_{+} \chi_{+}(x)=1+o\left(f^{n}\right) \text { all } n<\infty, \text { and } \\
& \phi_{+}^{\prime}(x) / \gamma_{+} \chi_{+}^{\prime}(x)=1+o\left(f^{n}\right) \text { all } n<\infty .
\end{aligned}
$$


Since the $\chi_{-}$terms are completely negligible there, the calculation of $\epsilon_{+}$and $\zeta_{+}$ is quite easy from (c.3) and (b.7)-(b.10), (b.26)-(b.28):

$$
\begin{aligned}
\epsilon_{+}= & -\pi f^{-1 / 3}(-\lambda)^{-1 / 4} \gamma_{+} T^{-1 / 2}(f) \exp \left(-\int_{-\lambda / f-f^{-\beta}}^{t_{2}}(C(x))^{1 / 2} d x\right) \\
& \cdot \frac{1}{\sqrt{\pi}} f^{1 / 6}(-\lambda)^{-1 / 4} \exp \left(\frac{2}{3} f^{1 / 2}\left(f^{-\beta}\right)^{3 / 2}\right)\left\{-2 \sqrt{-\lambda}+O\left(f^{3 \beta / 2-1 / 2}\right)\right\} .
\end{aligned}
$$

Thus

$$
\epsilon_{+}=2 \sqrt{\pi} f^{-1 / 6} \gamma_{+} T^{-1 / 2}(f) \cdot\left(1+O\left(f^{3 \beta / 2-1 / 2}\right)\right)
$$

where $\gamma_{+}$is given to $O\left(f^{\alpha}+f^{1-2 \alpha}\right)$ in (c.4). Just as with $\delta_{+}$, there is a cancellation in

$$
\begin{aligned}
\zeta_{+}= & \frac{\sqrt{\pi}}{2} f^{-1 / 6}(-\lambda)^{-1 / 2} \gamma_{+} T^{-1 / 2}(f) \exp \left(-\frac{2}{3} f^{1 / 2-3 \beta / 2}\right. \\
& \left.-\int_{-\lambda / f-f^{-\beta}}^{t_{2}}(C(x))^{1 / 2} d x\right) \cdot O\left(f^{3 \beta / 2-1 / 2}\right),
\end{aligned}
$$

and so

$$
\phi_{+}(x) / \epsilon_{+} a(x)=1+O\left(f^{3 \beta / 2-1 / 2}\right)
$$

uniformly on Region III (because, in terms of the Airy functions, on Region III,

$$
\begin{aligned}
& |b(x ; f, \lambda) / a(x ; f, \lambda)| \exp \left(-\frac{4}{3} f^{1 / 2-3 \beta / 2}\right) \\
& \quad \leqslant\left|b(x ; f, \lambda) \exp \left(-\frac{4}{3}\left(-f^{\frac{1}{3}}(x+\lambda / f)\right)^{\frac{3}{2}} / a(x ; f, \lambda)\right)\right|
\end{aligned}
$$

is bounded uniformly in $x$ as $f \rightarrow 0$ ).

Since $\eta_{+}$is the analytic continuation of $\phi_{+}$, the next boundary point is $x=-\lambda / f+e^{-\pi i / 6} f^{-\beta}$, where

$$
\begin{aligned}
& \eta_{+}(x) / \epsilon_{+} a(x)=1+o\left(f^{n}\right), \text { all } n<\infty, \text { and } \\
& \eta_{+}^{\prime}(x) / \epsilon_{+} a^{\prime}(x)=1+o\left(f^{n}\right), \text { all } n<\infty .
\end{aligned}
$$

The calculation of the coefficient $\theta_{+}$from $\epsilon_{+}$is essentially the inverse of the calculation of $\epsilon_{+}$from $\gamma_{+}$, and

$$
\theta_{+}=\gamma_{+} \cdot\left(1+O\left(f^{3 \beta / 2-1 / 2}\right)\right)
$$


There is again a leading-order cancellation in $\iota_{+}$, and, with (b.11),

$$
\begin{gathered}
\iota_{+}=\frac{1}{2} \epsilon_{+} W\left\{a, \chi_{+}\right\}_{\mid x=-\lambda / f+e^{-\pi i / \sigma_{f}-\beta}} \cdot\left(1+o\left(f^{n}\right)\right), \text { all } n ; \\
\iota_{+} \chi_{-}(x) / \theta_{+} \chi_{+}(x)=O\left(f^{3 \beta / 2-1 / 2}\right),
\end{gathered}
$$

uniformly in Region V. So

$$
\eta_{+}(x) / \gamma_{+} \chi_{+}(x)=1+O\left(f^{3 \beta / 2-1 / 2}\right)
$$

which completes the construction of the comparison functions $\phi_{+}$and $\eta_{+}$.

The construction of the other pair of comparison functions is quite similar, except that we start at $\infty$ and move in: Let

$$
\begin{aligned}
& \phi_{-}(x ; f, \lambda)= \begin{cases}\alpha_{-} \sigma_{+}(x)+\beta_{-} \sigma_{-}(x), & \text { Region I; } \\
\gamma_{-} \chi_{+}(x)+\delta_{-} \chi_{-}(x), & \text { Region II; } \\
\epsilon_{-} a(x)+\zeta_{-} b(x), & \text { Region III; }\end{cases} \\
& \eta_{-}(x ; f, \lambda)= \begin{cases}\kappa_{-} a(x)+\mu_{-} c(x), & \text { Region IV; } \\
\nu_{-} \chi_{+}(x)+\xi_{-} \chi_{-}(x), & \text { Region V }\end{cases}
\end{aligned}
$$

Then we solve for the coefficients starting with Regions V and III, where by definition from section 4 ,

$$
\begin{aligned}
& \xi_{-}=\frac{1}{\sqrt{\pi}} f^{1 / 6} T^{-1 / 2}(f) N(f) \\
& \nu_{-}=0 \\
& \zeta_{-}=N(f) \\
& \epsilon_{-}=0 .
\end{aligned}
$$

The normalization factor $N(f)$ is fixed by the condition that $W\left\{\phi_{-}, \phi_{+}\right\}=1$, so (4.9) for $N$ results from a comparison of (c.11) and (4.11c), (4.12c). Since the calculations are quite similar to the earlier ones, we will not do them in any detail, but note that the "right" coefficients $\beta_{-}, \delta_{-}, \zeta_{-}, \mu_{-}$, and $\xi_{-}$dominate as before, because of cancellations to leading order in the "wrong" coefficients and exponential dominance of one comparison function over the other throughout a given region. With this observation, the remaining dominant coefficients can be read off from the Wronskians of $\phi_{+}$with $\phi_{-}$and of $\eta_{+}$with $\eta_{-}$and our earlier 
calculations (c.4)-(c.14):

$$
\begin{aligned}
\mu_{-} & =\left(1 / \epsilon_{+} W\{c, a\}\right) \cdot\left(1+O\left(f^{\epsilon}\right)\right) \\
& =\left[\frac{f^{1 / 6}}{\sqrt{\pi}} \gamma_{+} T^{-1 / 2}(f) e^{\pi i / 6}\right]^{-1} \cdot\left(1+O\left(f^{\epsilon}\right)\right) ; \\
\delta_{-} & =\left(1 / \gamma_{+} W\left\{\chi_{-}, \chi_{+}\right\}\right) \cdot\left(1+O\left(f^{\epsilon}\right)\right)=\frac{1}{2 \gamma_{+}} \cdot\left(1+O\left(f^{\epsilon}\right)\right) ; \\
\beta_{-} & =\left(1 / W\left\{\sigma_{-}, \sigma_{+}\right\}\right) \cdot\left(1+O\left(f^{\epsilon}\right)\right)=\frac{1}{2 \sqrt{-\lambda}} \cdot\left(1+O\left(f^{\epsilon}\right)\right) .
\end{aligned}
$$

This completes the determination of $\phi_{-}$and $\eta_{-}$.

\section{REFERENCES}

1. M. Abramowitz and I. A. Stegun, Handbook of Mathematical Functions, N.B.S. Appl. Math. Ser. 55, Washington, National Bureau of Standards, 1964.

2. J. Avron AND I. HerbST, Spectral and scattering theory of Schrödinger operators related to the Stark effect, Commun. Math. Phys. 52 (1977), 239-254.

3. E. BalsLeV AND J. M. Combes, Spectral properties of many-body Schrödinger operators with dilation analytic interactions, Commun. Math. Phys. 22 (1971), 280-294.

4. L. BENASSI AND V. GRECCHI, Resonances in the Stark effect and strongly asymptotic approximations, J. Phys. B, to appear 1980.

5. C. Bender and T. T. Wu, Anharmonic oscillator, Phys. Rev. 184 (1969), 1231-1260.

6. _ Large order behavior of perturbation theory, Phys. Rev. Lett. 16 (1971), 461-465; Anharmonic oscillator, II. A study of perturbation theory in large order, Phys. Rev. D7 (1973), 1620-1636.

7. T. BANks, C. M. Bender AND T. T. Wu, Coupled anharmonic oscillators, I. Equal-mass case, Phys. Rev. D8 (1973), 3346-3366.

8. E. Brézin, J. C. LeGuillou, AND J. Zinn-Justin, Perturbation theory at large order, I. The $\phi^{2 N}$ interaction, Phys. Rev. D15 (1971), 1544-1557.

9. H. BuchHolz, The Confluent Hypergeometric Function, New York, Springer, 1969.

10. J. M. Combes, An algebraic approach to quantum scattering, unpublished manuscript, 1971.

11. W. Y. CRUTCHFIELD II, Method for Borel-summing instanton singularities introduction, Phys. Rev. D19 (1979), 2370-2384.

12. R. J. Damburg AND V. V. Kolosov, An asymptotic approach to the Stark effect for the hydrogen atom, J. Phys. B11 (1978), 1921-1930.

13. P. S. Epstein, Zur Theorie des Starkeffektes, Ann. der Physik 50 (1916), 489-520.

14. The Stark effect from the point of view of Schroedinger's quantum theory, Phys. Rev. 28 (1926), 695-710.

15. N. Fröman and P.-O. Fröman, JWKB Approximation, Contribution to the Theory, Amsterdam, North-Holland, 1965.

16. G. Gamow, Constitution of Atomic Nuclei and Radioactivity, Oxford, Oxford University Press, 1931.

17. I. S. Gradshteyn AND I. M. Ryzhik, Table of Integrals, Series, and Products, New York, Academic Press, 1965. 
18. S. Graffi AND V. Grecchi, Resonances in Stark effect and perturbation theory, Commun. Math. Phys. 62 (1979), 83-96.

19. Confinement of the resonances in hydrogen Stark effect, J. Phys. B12 (1979), L 265-267.

20. S. Graffi, V. Grecchi, S. Levoni, AND M. Maioli, Resonances in one-dimensional Stark effect and continued fractions, J. Math. Phys. 20 (1979), 685-690.

21. S. Graffi, V. Grecchi, AND B. Simon, Complete separability of the Stark effect in hydrogen, J. Phys. A, 12 (1979), L193-L195.

22. E. Harrell, On the rate of asymptotic eigenvalue degeneracy, Commun. Math. Phys. 60 (1978), 73-95; The band-structure of a one-dimensional, periodic system in a scaling limit, Ann. Phys. 119 (1979), 351-369.

23. I. HerbSt, Unitary equivalence of Stark Hamiltonians, Math. Z. 155 (1977), 55-71.

24. - Dilation analyticity in constant electric field, I: The two body problem, Commun. Math. Phys. 64 (1979), 279-298.

25. I. Herbst ANd B. Simon, Stark effect revisited, Phys. Rev. Lett. 41 (1978), 67-69.

26. - Dilation analyticity in constant electric field, II: The N-body problem, Borel summability, submitted to Commun. Math. Phys.

27. J. Howland, Spectral concentration and virtual poles, Amer. J. Math. 91 (1969), 1106-1126.

28. C. G. J. JACOBI, Vorlesungen über Dynamik, Berlin, G. Reiner, 1884.

29. T. Kato, On the convergence of the perturbation method, J. Fac. Sci. Univ. Tokyo 6 (1951), 145-226.

30. C. Lanczos, Zur Theorie des Starkeffektes in hohen Feldern, Z. für Physik 62 (1930), 518-544; Zur Verschiebung der Wasserstoffterme in hohen elektrischen Feldern, Z. für Physik 65 (1930), 431-455; Zur Intensitätsschwächung der Spektrallinien in hohen Feldern, Z. für Physik 68 (1931), 204-232.

31. L. D. Landau and E. M. Lifshitz, Quantum Mechanics, New York Pergamon Press, 1977.

32. R. E. LANGER, The asymptotic solutions of ordinary linear differential equations of the second order, with special reference to a turning point, Trans. Amer. Math. Soc. 67 (1949), 461-490.

33. R. Lavine, "Spectral density and sojourn times," in: Atomic Scattering Theory, J. Nuttall, ed., London, Ontario; Univ. of Western Ontario Press, 1978.

34. L. N. Lipatov, Divergence of the perturbation theory series and pseudo-particles, Soviet Phys. JETP Lett. 25 (1977), 104-107.

35. J. J. Loeffel and A. Martin, Propriétés Analytiques des Niveaux de l'Oscillateur Anharmonique et Convergence des Approximants de Padé, Proc. R.C.P. 25, May, 1970.

36. J. J. Loeffel, A. Martin, B. Simon, AND A. Wightman, Padé approximants and the anharmonic oscillator, Phys. Lett. 30B (1969), 656-658.

37. C. Lovelace, "Three-particle systems and unstable particles" in: Strong Interactions and High Energy Physics, Edinburgh, Oliver and Boyd, 1964.

38. J. R. OPPENHEIMER, Three notes on the quantum theory of aperiodic effects, Phys. Rev. 31 (1928), 66-81.

39. M. Reed AND B. Simon, Methods of Modern Mathematical Physics IV. Analysis of Operators, New York, Academic Press, 1978.

40. T. Regge and V. De Alfaro, Potential Scattering, Amsterdam, North-Holland, 1965.

41. E. Schrödinger, Quantisierung als Eigenwertproblem, III., Ann. der Physik 80 (1926), 457-490.

42. K. Schwarzschild, Zur Quantenhypothese, Sitzungsber. der kön. preuss. Akad. der Wiss. 25 (1916), 548-568.

43. J. SCHWInger, Field theory of unstable particles, Ann. Phys. 9 (1960), 169-193.

44. A. J. F. Siegert, On the Derivation of the Dispersion Formula for Nuclear Reactions, Phys. Rev. 56 (1939), 750-752.

45. H. J. Silverstone, Perturbation theory of the Stark effect in hydrogen to arbitrarily high order, Phys. Rev. A18 (1978), 1853-1864.

46. Asymptotic relations between the energy shift and ionization rate in the Stark effect in hydrogen, Johns Hopkins Univ., preprint. 
47. H. J. Silverstone, B. G. Adams, J. Čížek, AND P. Otto, Asymptotic formula for the perturbed energy coefficients and calculation of the ionization rate by means of high-order perturbation theory for hydrogen in the Stark effect, Johns Hopkins Univ. and Univ. of Waterloo preprint.

48. B. Simon, Coupling constant analyticity for the anharmonic oscillator, Ann. Phys. 58 (1970), 76-136.

49. - Resonances in $N$-body quantum systems with dilation analytic potentials and the foundations of time-dependent perturbation theory, Ann. Math. 97 (1973), 247-274.

50. - Phase space analysis of simple scattering systems: Extensions of some work of $V$. Enss, Duke Math. J. 46 (1979), 119-168.

51. — Functional Integration and Quantum Physics, New York, Academic Press, 1979.

52. T. SPENCER, in prep.

53. J. STARK, Beobachtungen über den Effekt des electrischen Feldes auf Spektrallinien, Sitzungsber. der kön, preuss. Akad. der Wiss. 47 (1913), 932-946.

54. E. C. TItchmarsh, Eigenfunction Expansions Associated with Second Order Differential Equations, Part II, Oxford Univ. Press, 1958.

55. - Some theorems on perturbation theory, I-V, Proc. Roy. Soc. A 200 (1949), 34-46; A 201 (1950), 473-479; A 207 (1951), 321-328; A 210 (1951), 30-47; J. Analyse Math. 4 (1954-1956), 187-208; Eigenfunction expansions associated with partial differential equations, V, Proc. London Math Soc. 5 (1955), 1-21.

56. K. Veselić ANd J. Weidmann, Potential scattering in a homogeneous electric field, Math. Z. 156 (1977), 93-104.

57. K. YajIma, Spectral and scattering theory for Schrödinger operators with Stark field. I, J. Fac. Sci. Univ. Toyko, to appear. II. Univ. of Virginia preprint.

58. T. Yamabe, A. TAchibana, AND H. J. Silverstone, Theory of the ionization of the hydrogen atom by an electrostatic field, Phys. Rev. A 16 (1977), 877-890.

59. — - Perturbation theory of resonant states induced by an electrostatic field: One dimensional model, J. Phys. B 10 (1977), 2083-2100.

60. B. Simon, Complex scaling; A rigorous overview, Int. J. Quant. Chem. 14 (1978), 529-542.

61. J. Howland, Puiseux series for resonances at an embedded eigenvalue, Pac. J. Math. 55 (1974), 157-176.

62. I. HerbSt, Exponential decay in the Stark effect, Commun. Math. Phys., to appear.

Harrell: Department of Mathematics, The Johns Hopkins University, Baltimore, MARYLAND 21218

Simon: Departments of Mathematics and Physics, Princeton University, Princeton, New JERSEY 08540 